\title{
Review Article \\ Glioma Stem Cells and Their Microenvironments: Providers of Challenging Therapeutic Targets
}

\author{
Elena Codrici, ${ }^{1}$ Ana-Maria Enciu, ${ }^{1,2}$ Ionela-Daniela Popescu, ${ }^{1}$ \\ Simona Mihai, ${ }^{1}$ and Cristiana Tanase ${ }^{1}$ \\ ${ }^{1}$ Biochemistry-Proteomics Department, Victor Babes National Institute of Pathology, Sector 5, 050096 Bucharest, Romania \\ ${ }^{2}$ Cell Biology and Histology Department, Carol Davila University of Medicine and Pharmacy, Sector 5, 050474 Bucharest, Romania \\ Correspondence should be addressed to Elena Codrici; raducan.elena@gmail.com
}

Received 4 September 2015; Revised 12 November 2015; Accepted 6 January 2016

Academic Editor: Justin D. Lathia

Copyright (C) 2016 Elena Codrici et al. This is an open access article distributed under the Creative Commons Attribution License, which permits unrestricted use, distribution, and reproduction in any medium, provided the original work is properly cited.

\begin{abstract}
Malignant gliomas are aggressive brain tumors with limited therapeutic options, possibly because of highly tumorigenic subpopulations of glioma stem cells. These cells require specific microenvironments to maintain their "stemness," described as perivascular and hypoxic niches. Each of those niches induces particular signatures in glioma stem cells (e.g., activation of Notch signaling, secretion of VEGF, bFGF, SDF1 for the vascular niche, activation of HIF $2 \alpha$, and metabolic reprogramming for hypoxic niche). Recently, accumulated knowledge on tumor-associated macrophages, possibly delineating a third niche, has underlined the role of immune cells in glioma progression, via specific chemoattractant factors and cytokines, such as macrophage-colony stimulation factor (M-CSF). The local or myeloid origin of this new component of glioma stem cells niche is yet to be determined. Such niches are being increasingly recognized as key regulators involved in multiple stages of disease progression, therapy resistance, immune-escaping, and distant metastasis, thereby substantially impacting the future development of frontline interventions in clinical oncology. This review focuses on the microenvironment impact on the glioma stem cell biology, emphasizing GSCs cross talk with hypoxic, perivascular, and immune niches and their potential use as targeted therapy.
\end{abstract}

\section{Introduction}

Gliomas, representing tumors of astroglial origin, have been classified by World Health Organization (WHO) into four grades of ascending malignancy according to the histological criteria. Presenting one of the highest mortality rates, glioblastoma multiforme (GBM, WHO grade IV) only benefits from palliation as far as conventional therapy goes. In spite of intensive efforts and the progress achieved in tumor biology and clinical treatment, little improvement of the average survival for a newly diagnosed GBM patient to less than 15 months was recorded [1]. Both GBM biology in general and the cellular origin of this disease in particular are not fully understood, thus restraining clinical advances. Vascular endothelial proliferation appears to be a highly angiogenic tumor in GBM, since extensive blood vessel growth is essential for tumor progression and invasion [2]. The vasculature is associated with GBM, reducing hypoxia; it is generally required for tumor survival. The cancer stem cell hypothesis suggests that all cancer types are comprised of a subset of highly aggressive cells. These propagate and preserve the tumors thought to have unlimited self-renewal capacity and potent tumorigenicity [3].

GSCs and normal neural stem cells (NSCs) present similar properties, such as the expression of neural stem cell markers, infinite self-renewal and long-term proliferation ability, neurospheres formation, and multipotential differentiation capacity $[3,4]$. Furthermore, according to in vivo evidence, GSCs can initiate highly invasive tumors [5]. GSCs have been proven to be resistant to various chemotherapeutic agents, such as temozolomide, the standard chemotherapeutic agent for GBM treatment, allowing these cells to survive therapy, leading to disease recurrence [6-8].

It has been recently demonstrated by experimental studies that GSCs are enriched in specific niches around tumor vessels and areas of necrosis, the latter associated 
with restricted oxygen levels. Hence, GSCs display a symbiotic relationship with perivascular/proliferative and hypoxic/ perinecrotic niches [8-10]. Endothelial cells (ECs) generate numerous growth factors that fuel GSC self-renewal, tumorigenicity, and survival [11-13]. GSCs may transdifferentiate into endothelial cells or pericytes, forming their own vascular niches [14-17]. The capacity of GSCs to transdifferentiate into functional endothelial cells is still under debate. While conventional theory suggests that GBM tumor vasculature derives from existing vessels or from bone marrow progenitor cells, there are recent studies that sustain the hypothesis that a large subset of endothelial cells can be generated by GSCs [18].

Many cytokines and chemokines are produced by GSCs as well, some of which are known to activate endothelial cells [19-21]. According to this hypothesis, GSCs may in turn regulate the tumor vasculature and, consequently, the extent of tumor angiogenesis.

The main focus of this review will be on the interaction between GSCs and their microenvironment, emphasizing the molecular processes through which GSCs cross-talk with hypoxic and vascular niches. Another key point will be the interaction of GSCs with tumor-infiltrating immune cells and the role of GSCs in the regulation of tumor angiogenesis in GBM.

\section{Glioma Stem Cells (GSCs) and Their Markers}

Infinite self-renewal, unlimited proliferative potential, multilineage differentiation capacity, neurospheres formation, and expression of neural stem cell markers (e.g., CD133/ prominin-1, Sox2, and Nestin) represent some of the specific features of GSCs $[22,23]$. Other various candidate markers that are used in order to enrich GSCs have been discovered over the last ten years, among which are CD44 [24], CD49f (integrin a6) [25], Musashi [26], Nestin [27, 28], Nanog [29$31]$, Oct4 [29, 30, 32], and Sox2 [33, 34]; nevertheless, the quest for a universal GSCs marker continues [4].

GSCs seem to be genuine cancer reservoirs; consequently, any therapy approach aiming at brain cancers is obstructed by the resistance to treatment that these cells show, since GSCs are capable of whole tumor regeneration once the treatment has concluded $[35,36]$.

Lathia et al. demonstrated that GSCs constitute the origin and source of tumor recurrence in glioblastoma $[7,8]$, by injecting differentially labeled GSCs and non-GSCs into mice. The only fraction to produce tumors was the GSC, despite representing only $10 \%$ of the implanted cells [8]. It appears that the ones responsible for tumor growth are GSCs, rather than the more differentiated tumor cells. In addition, the former are also involved in tumor recurrence following drug resistance. Chen et al. showed that when temozolomide treatment is interrupted in a spontaneous murine glioma model, Nestin-positive GSC population is the first cell population that undergoes proliferation and leads to tumor regrowth [7]. GSCs have been found to be enriched in recurrent gliomas [37, 38]. When isolating GSCs from recurrent tumors, they generate more aggressive invasive tumors in athymic mice than when isolated from primary tumors derived from the same patient [38]. Subsequently, it seems that GSCs contribute to tumor regrowth from minimal residual disease after surgery. GSCs display great resistance to chemotherapeutic agents, as well as a highly invasive feature [39]. Another property of GSCs in terms of resistance is their particular resistance to radiation, in comparison with the more differentiated glioma cells, with this being the consequence of an effective DNA damage repair response [35]. Notch signaling in GSCs promotes self-renewal, protects against radiation, and represses differentiation [40].

Research has focused particularly on the identification of intrinsic molecular pathways involved in the regulation of GSCs features, such as stemness and tumorigenicity, ever since the GSCs have been identified [5, 41, 42] (Table 1).

2.1. CD133 Controversy. The identification of specific surface markers is necessary in order to isolate GSCs and subsequently characterize them for future GSC-targeted therapies [90]. CD133 (prominin-1) is one of the earliest stem-cell surface markers used for identification and isolation of cancer stem cells in malignant brain tumors. Singh et al. successfully isolated a CD133+ cell subpopulation from human brain tumors that exhibited stem cell properties in vitro. They reported the development of a xenograft that identified human brain tumor initiating cells that initiate tumors in vivo. Only the CD133+ brain tumor cells could initiate tumor in mouse brain, whereas injection of CD133- cells did not lead to tumor formation [22].

However, accumulated results in GBM molecular research led to several CD133 related controversies. For example, GSCs display a variation in the levels of CD133 expression that did not directly correlate with the tumorigenic potential [91]. Most importantly, different studies suggested that CD133- tumor cells isolated from GBMs can also be stably cultured under stem cell conditions. Similar to the CD133+ cells, these cells also showed "stem cell" properties such as self-renewal, differentiation in vitro, and formed transplantable tumors in a xenograft model $[92,93]$. Further phenotypic analysis showed that unlike the CD133+ cells, which can form floating spheroids in culture, the CD133- cells tend to grow as adherent spheres. This observation led to the assumption that CD133+ and CD133cells may originate from different pools of self-renewing glioma stem cells (GSCs) [94]. It has recently been reported that a small population of CD133- cells can give rise to CD133+ cells, suggesting a possible stem cell hierarchy in the spheroid culture system that may or may not have in vivo relevance [95]. These results, however, have been brought into discussion in 2013 by Brescia et al., who argued that the + or - CD133 status depends, in fact, on the protein subcellular localization between the cytoplasm and the plasma membrane [96].

Data continues to accumulate, however, on CD133 biology, as it has been repeatedly demonstrated to be essential for GSC maintenance and neurosphere formation [96] and it is a good indicator of resistance to conventional therapies [36]. 


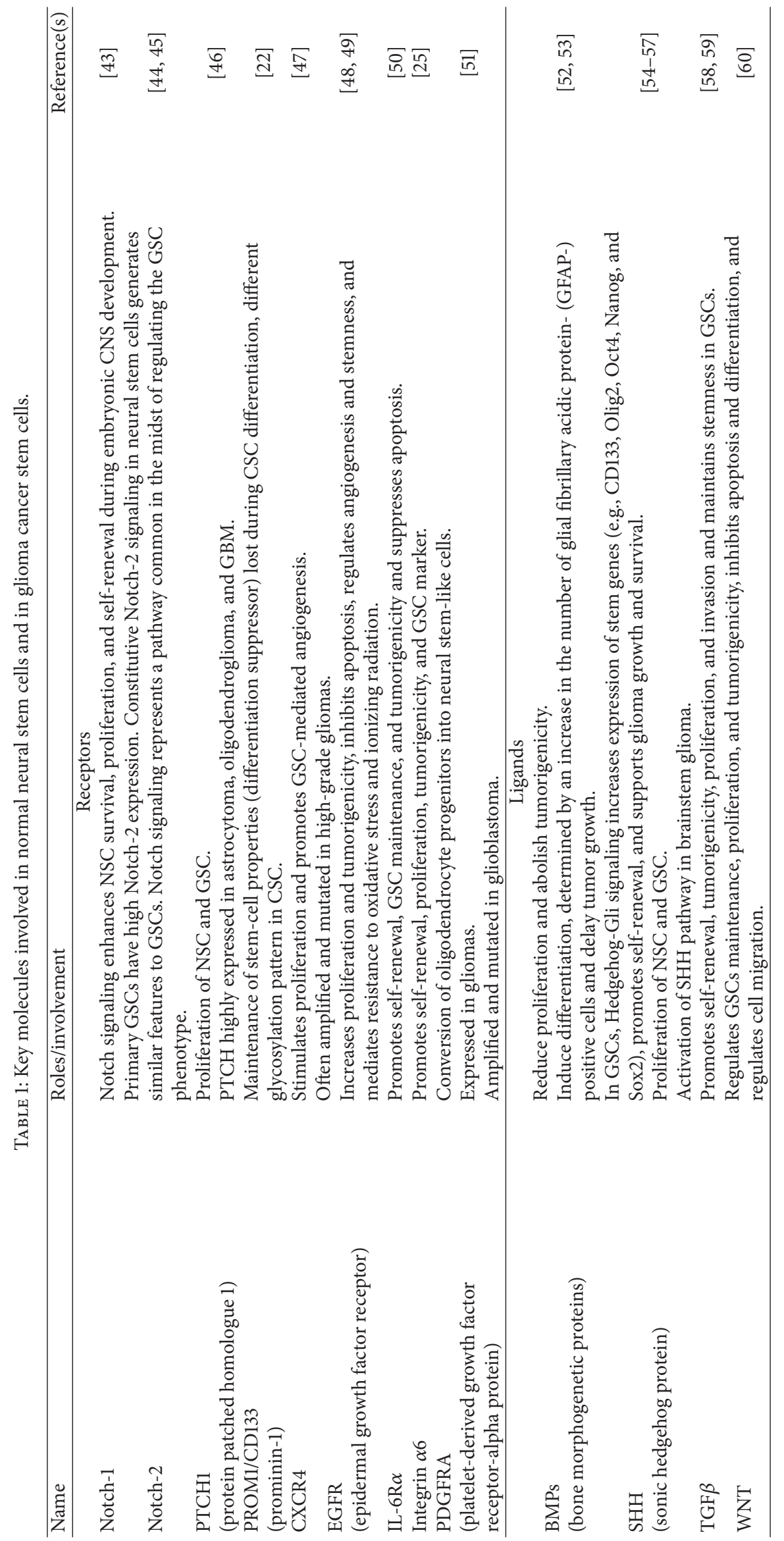




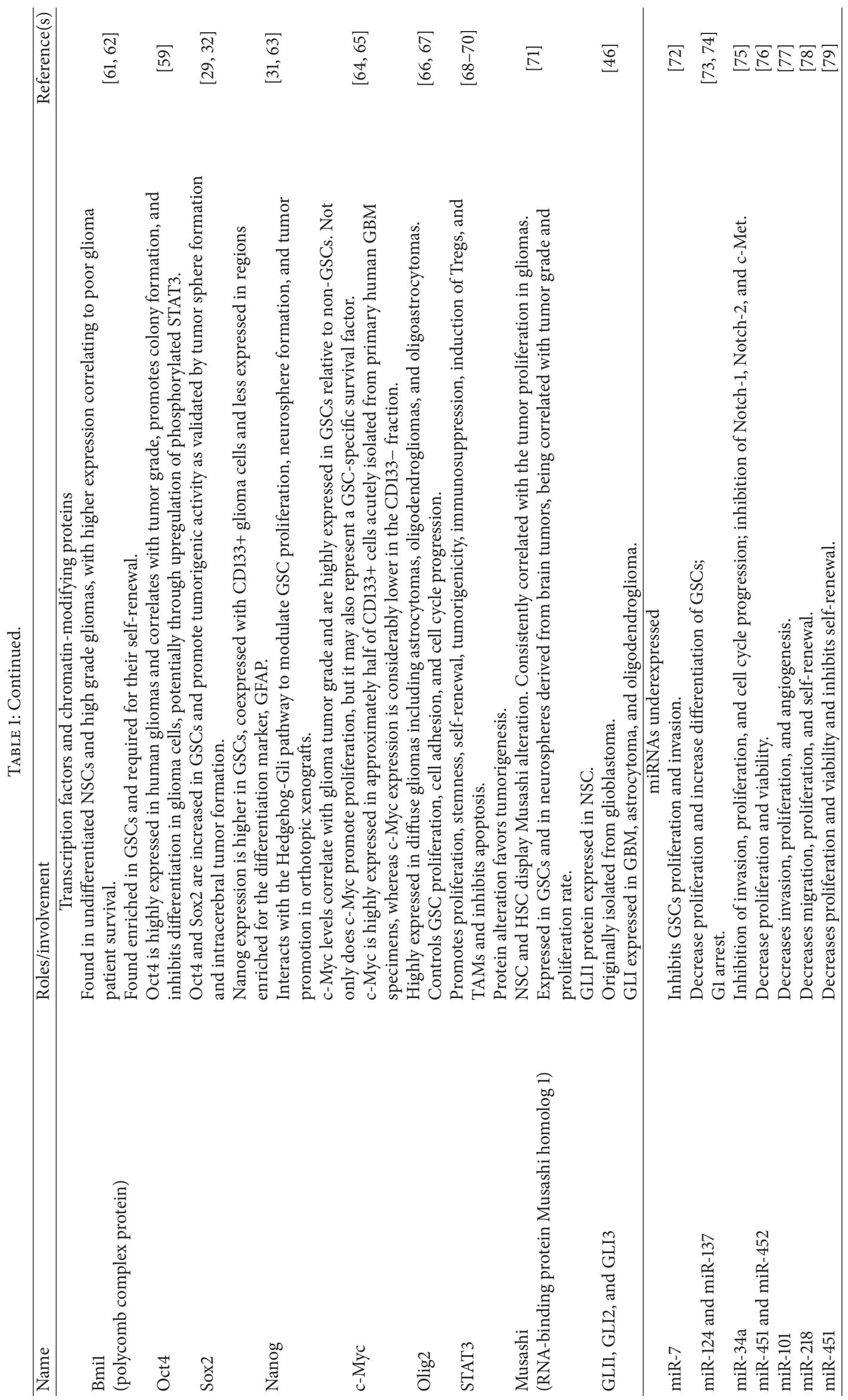




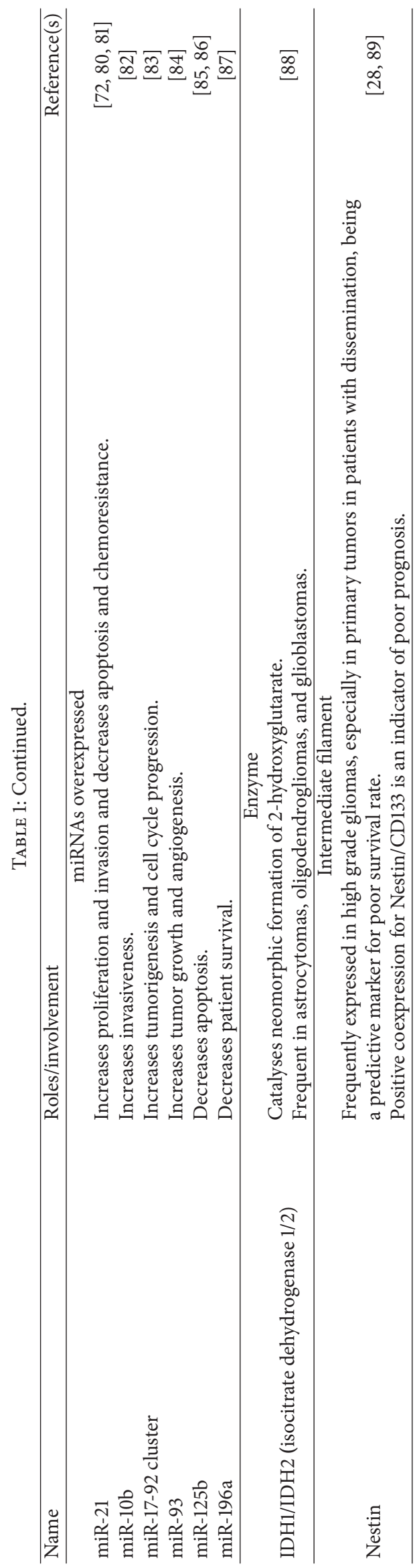


Moreover, the association of CD133 with other markers could enhance the potential pathological prognostic markers for glioma patients [97]. For instance, the association of coexpression of Nestin/CD133 is helpful in predicting the aggressive nature of gliomas [28]. The presence of CD133+/Ki67+ positive cells may be an indicator of tumor progression and unfavorable prognosis [98].

2.2. Intratumor Heterogeneity of GSCs. Yet another factor to be added to the difficult task of GSCs characterization is the heterogeneity of their own population. At least two phenotypes of GSCs (proneural and mesenchymal phenotypes) have been reported [99], each characterized by a different transcriptional profile [100] and different metabolism [101].

Single cell-derived clones of human glioblastoma tumors with stem properties (e.g., able to reconstitute the original tumor) exhibited functional and morphologic heterogeneity. Even though in vitro all clones displayed neuronal precursor phenotype, individual clone-derived populations expressed different GBM markers (such as EGFR, EGFRvIII, and PTEN) and clone by clone variability in response to multiple drugs [102].

Further on, heterogeneity arises with microenvironment change. If in vitro nonproliferating and proliferating cells of the parental tumor showed no significant differences in their transcriptional profiles, in vivo clonal orthotopic tumors derived from proliferative cells upregulated distinct sets of genes, when compared with their nonproliferative counterparts [103].

A functional consequence of transcriptional and metabolic heterogeneity is the frequency of self-renewal and differentiation rate of progenitor cells. The question whether GSC heterogeneity is maintained during repetitive cycles of self-renewal or lost to those clones with high frequency cell division has been recently answered by Sugimori et al. They reported that "the growth characteristics of GSs are retained during repopulation ... and do not support the clonal evolution model, at least not with regard to SC heterogeneity." It seems that, in order to recapitulate over generations, the heterogeneity of the initial population, at least in terms of proliferative activity, cancer stem cells must exhibit plasticity, meaning that "clones change their spatial and temporal properties" [104].

\section{GSCs and Their Microenvironment (Niches)}

Stem cells and these niches do not display a passive relationship; they have a dynamical interaction with their microenvironment. While stem cells actively influence their microenvironments, they are regulated by signaling from that same microenvironment. Likewise, GSCs also exist in specific niches that play a role in enhancing the stem-like features of GSCs, promote invasion and metastasis of GSCs, and even affect response to therapy/escape from therapy. It is essential to understand the bidirectional cross talk between GCSs and the niches in order to disclose the role of this controversial population in GBM initiation, progression, invasion, and therapeutic resistance.
3.1. The Perivascular/Proliferative Niche. In perivascular regions, GSCs appear to be enriched, where a great deal of regional signals have been found to promote their phenotypes [105]. GSCs are generally located near the endothelial cells (ECs) that line capillaries, especially in the subventricular zone and the hippocampus $[106,107]$.

\subsubsection{Components of Vascular Niche}

(1) Soluble Factors: Origin and Effect on GSCs. It has been reported that GSCs release high levels of proangiogenic factors, such as vascular endothelial growth factor (VEGF) that drives the migration of newly EC into the mass and promotes angiogenesis. ECs overexpress VEGF receptors (VEGFR2); thus, an environment of high VEGF increased ECs proliferation, migration, and blood vessel permeability. Permeability alterations are associated with increased edema usually observed in GBM [106].

Moreover, Sonic Hedgehog $(\mathrm{SHH})$ is considered one of the central soluble factors secreted by ECs that promote the acquirement of CSC properties by activating the $\mathrm{HH}$ signaling pathway. GSCs display active SHH-GLI1 signaling and regulate GSC self-renewal and glioma growth $[46,108]$. In primary glioma samples, GSCs CD133+ are found in the area near SHH-expressing ECs. Tumor sphere formation and the expression of stemness-related molecules are promoted by ECs through glioma associated oncogene homologue 1 (GLI1) enhancement and its translocation from the cytoplasm to the nucleus [109].

Tumor ECs expressed SHH [110] in a PDGF-driven mouse glioma model, providing a potential mechanism for GLI1 activation in GSCs.

It has been recently found that the secretion of FGF2 by GBM cells enhances the blood brain barrier function of ECs, which also contributes to drug resistance in GBM [111]. Survivin, an angiogenesis-promoting protein, could activate the release of FGF-2, along with VEGF, in gliomas and thereby stimulate an increase in growth and proliferation in the tumors [112]. FGF-2 helps maintain especially GSCs stemness. When removed from GSCs lines, it resulted in differentiation; this was not observed when the cells were in the presence of the growth factor [113]. FGF-2 is effective at inducing Nestin, in C6 glioma cells, proving its contribution to the stemness of glioma cells [114]. Autocrine production of FGF-2 in combination with EGF may also be responsible for retaining the self-renewal potential of GSCs [115]. FGF2's role in GSCs remains to be characterized. The therapies targeting FGF-2 might be effective at destroying GSCs, since the growth factor is important in preserving the stemness feature of GSCs [116].

Osteopontin, which is derived from the perivascular niche, promotes GSCs phenotype by activating CD44, one of the CSC markers. The C-terminal intracellular domain of CD44 is essential for inducing GSCs characteristics by enhancing the function of hypoxia inducible factor $2 \alpha$ (HIF-

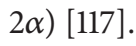

Besides the above-mentioned factors, GSCs secrete other proangiogenic growth factors as well. When comparing the proteomes of four different GSCs with four normal 


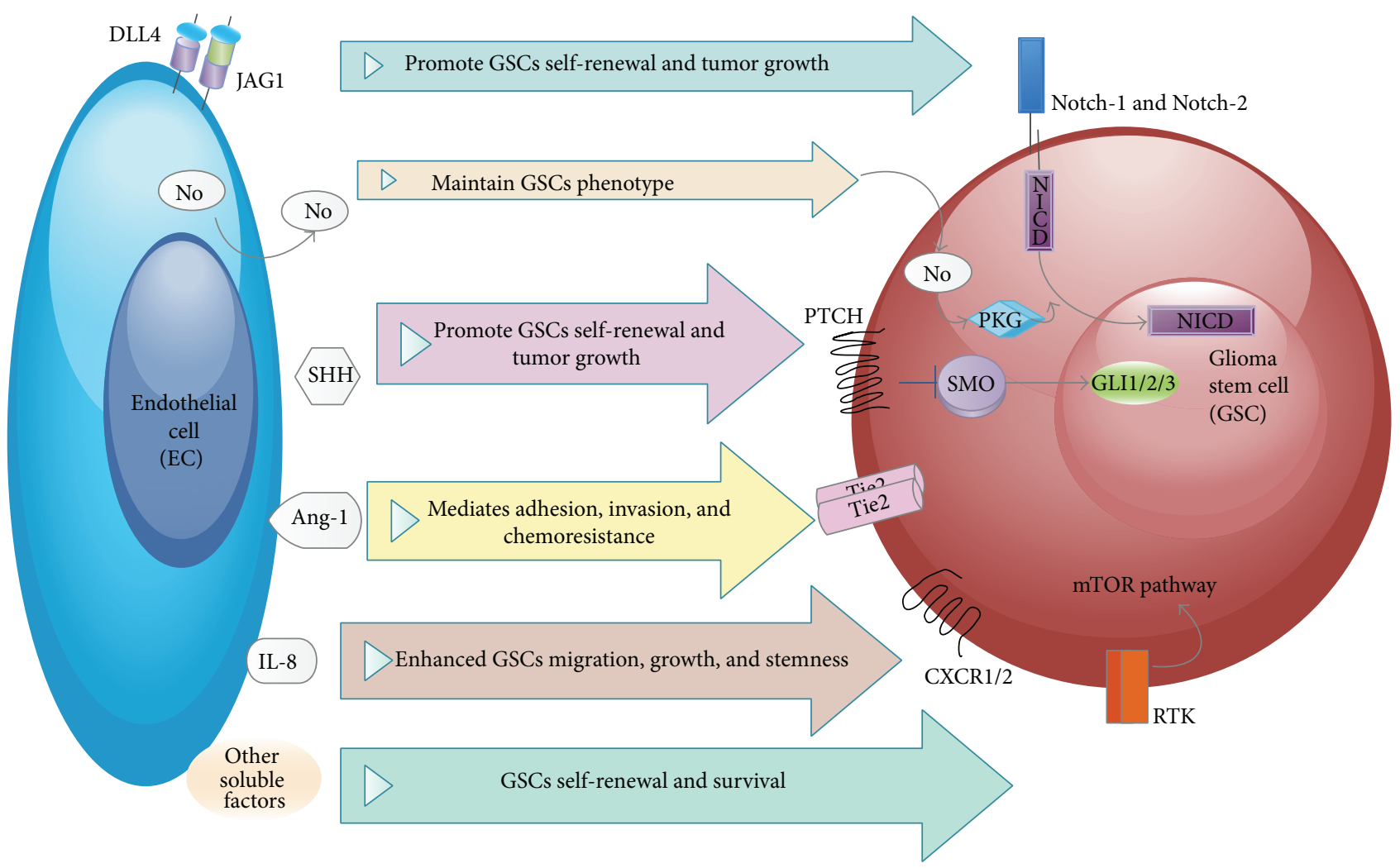

FIGURE 1: Effects of endothelial cells on GSCs. ECs produce membrane-bound Notch ligands Jagged-1 (JAG1) and Delta-like 4 (DLL4) that bind to Notch receptors on GSCs and promote GSCs self-renewal and tumor growth; nitric oxide (NO) that maintains GSCs phenotype; ligand sonic hedgehog ( $\mathrm{SHH}$ ) that promotes GSCs self-renewal and tumor growth; angiopoietin-1 (Ang-1) that mediates adhesion, invasion, and chemoresistance; $I L-8$ that enhanced GSCs migration, growth, and stemness; other soluble factors that stimulate GSCs self-renewal and survival.

NSCs cultures, the levels of HDGF (hepatoma-derived growth factor) were found twofold higher in GSCs. By further analyzing the GSC conditioned medium, it has been revealed that only GSCs secrete HDGF, which promotes endothelial cell migration in vitro and angiogenesis in a subcutaneous in vivo model [21]. Identifying a specific angiogenic factor by GSCs as compared to normal stem cells allows selective targeting of tumor angiogenesis without affecting the normal stem cell pool. This proves particularly significant data, suggesting that normal stem cells produce cytokines (e.g., BMP7), which act as suppressors of GSC activity [118].

An essential growth factor expressed in GBM is growth hormone releasing hormone (GHRH) [119]. It causes increased tumor cell proliferation, migration, and tumor progression. GHRH may also play a role in the activation of stromal fibroblasts in the tumor microenvironment by regulating $\alpha$-SMA expression. It remains to be elucidated whether GHRH specifically affects GSCs and its effects on tumor endothelial cells [120].

(2) Cells: Cell to Cell Interactions. The interaction between GSCs and ECs promotes activity in critical stem pathways, such as Notch signaling. GSCs Nestin-positive cells express the Notch receptors Notch-1 and Notch-2 and show elevated level of Notch activity [11]. ECs express the Notch ligands Delta-like 4 (DLL4) and Jagged-1. Knockdown of these ligands in brain microvascular endothelial cells (BMECs) reduced tumor growth upon cotransplantation of GSCs with BMECs [11]. GSCs may directly stimulate the expression of Notch ligands on ECs suggested by the findings that GSCs secrete elevated levels of VEGF [19], which induces DLL4 expression in ECs [121, 122] (Figure 1).

EC-derived nitric oxide (NO) can activate Notch signaling pathway in GSCs as well through NO/cGMP/PKG; therefore, it promotes the stem cell phenotype $[123,124]$. GSCs produce NO endogenously, which supports GSC growth and tumorigenicity [124]. Endothelial nitric oxide synthase (eNOS) also produces NO in the tumor vasculature. Upon loss of eNOS, it suppresses Notch signaling in vivo, it delays glioma genesis, and it prolongs the survival of tumor-bearing mice [123].

Furthermore, combined treatment of CSCs and vascular niches should not be overseen. Following radiation, Jagged-1, the ligand for Notch, was shown to be increased in ECs [125]. This suggests that Notch signaling is critical for ECmediated radioresistance of CSCs [126].

(3) Extracellular Matrix. The perivascular region is also enriched for extracellular matrix proteins (e.g., laminin) 
that are capable of promoting proliferation, survival, and migration of GSCs. GSCs are enriched for integrin $\alpha 6$ [25], acting as a receptor for laminin in complex with integrin $\beta 1$ or $\beta 4$. The integrin $\alpha 6$-expressing cell population is localized in the perivascular compartment of human GBM and silencing of integrin $\alpha 6$ reduces the self-renewal and tumorigenicity of glioblastoma cells. Likewise, adult NSCs, which are closely apposed to the laminin-containing extracellular matrix surrounding vascular endothelial cells, express $\alpha 6 / \beta 1$ integrin and its blockade inhibits neural stem cell adhesion to endothelial cells [127]. Integrins $\alpha 6-\beta 1$ also play a cytoprotective role for ECs by increasing expression of antiapoptotic proteins, such as cFLIP, and inducing the prosurvival of the TNF $\alpha$ pathway [128].

The interaction between vascular niche and GSCs also involves chemokines and their receptors. CXCR4 works as a biomarker of CSCs in several types of cancer, including glioma [129]. CXCR4-positive tumor cells can selfrenew in a serum-free medium and display potent tumorinitiating capability. The ligand for CXCR4, namely, CXCL12, is secreted by ECs and the immune cells in tumor microenvironment [130], which highlights the importance of CXCL12/CXCR4 axis in the maintenance of GSCs in vascular niches. By using a three-dimensional culture system, Infanger et al. proved that ECs promoted GSC-like properties by secreting enhanced levels of the chemokine CXCL8/IL-8 and upregulating its cognate receptors CXCR1 and CXCR2 [12]. According to these results, chemokine signaling is involved in vascular niches stemness regulation of GSCs.

3.1.2. The Role of Perivascular Niche in GSCs Biology. Perivascular niche appears to play a role in promoting the radioresistance of brain tumor CSCs. Due to their ability to activate the $\mathrm{PI} 3 \mathrm{~K} / \mathrm{Akt} / \mathrm{mTOR}$ pathway and undergo transient, PTEN and p53-dependent cell cycle arrest, CSCs localized in the vicinity of blood vessels in the brain were resistant to radiation. Inhibition of Akt signaling sensitized perivascular CSCs to radiation-induced apoptosis [131]. Inhibition of Notch signaling with gamma-secretase inhibitors (GSIs) impaired radiation-induced Akt activation and increased radiosensitivity of glioma stem cells. Knockdown of Notch-1 or Notch-2 sensitized glioma stem cells to radiation. The radioprotective functions of Notch were specific for GSCs but not non-GSCs [40].

ECs of vascular niches are crucial for inducing chemotherapy resistance of GSCs. Nonetheless, according to recent studies, mural cells of vascular niches also played a role in the induction of drug resistance of GSCs. The protective role of ECs and mural cells in GSCs resistance against radio/chemotherapy emphasizes the importance of vascular niche in targeted cancer therapy.

3.1.3. GSCs Can Shape the Perivascular Niche. GSC-derived factor stimulates the ECs proliferation, angiogenesis; GSCs recruit endothelial progenitor cells from bone marrow and GSCs transdifferentiation into pericytes.

Pericyte recruitment is induced by ECs release signals [132]. Pericytes secrete growth factors that stimulate ECs proliferation and proteases that contribute to the modulation of the surrounding extracellular matrix and guide ECs migration [133]. The resulting pericyte coverage is crucial for vessel remodeling, maturation, and stabilization and has been involved in therapeutic resistance in tumors. Direct contact establishes reciprocal communication between ECs and pericytes, either by paracrine signaling or by a newly described chemomechanical signaling pathway [134]. Signaling molecules such as angiopoietin-1/2 and Tie2 (Ang/ Tie2), transforming growth factor- $\beta$ (TGF- $\beta /$ TGF- $\beta$ R), and platelet-derived growth factor- $\beta$ (PDGF $\beta$ /PDGFR- $\beta$ ), which are related to EC viability, mural cell differentiation, and pericyte recruitment, respectively, are involved in the cross talk coordination [135].

Based on the work of Ricci-Vitiani et al., it appears that part of the vasculature in GBM originates from tumor cells. They analyzed the vasculature in 15 human glioblastoma patient samples and found that a large subset of endothelial cells harbored the same mutations and chromosomal aberrations as the tumors themselves. They also showed that in vitro culture of GSCs in endothelial conditions generated progeny with phenotypic and functional features of endothelial cells. Subcutaneous injection of GSCs in immunocompromised mice produced tumor xenografts; the tumor vessels were composed of human endothelial cells. All these findings describe a new mechanism for tumor vasculogenesis and may explain the presence of cancer-derived endothelial-like cells in several malignancies [15].

Various other studies have recently explored the phenomenon of tumor-derived vasculature in GBM. Recent reports found very similar results to those of Ricci-Vitiani et al., showing that oncogene induced glioblastoma tumors gave rise to tumor-derived endothelial cells, as indicated by GFP expression. These studies also found that a subpopulation of endothelial cells within tumors harbored the same genetic signature as the tumor itself $[14,136]$. Chiao et al. reported that GCSs formed vasculogenic mimicry in tumor xenografts and expressed provascular molecules [137].

However, Rodriguez et al. mention that "while the potential of stem-like cancer cells to form endothelium in culture seems clear, in our clinical experience using a variety of molecular markers, neoplastic cells do not contribute significantly to the endothelial-lined vasculature of primary human glioblastoma." At the end of the study, their observations were that glioblastoma cells incorporated into tumor vessels appear rather unfrequent, and thus it is of questionable clinical or therapeutic significance [138].

Interestingly, Cheng et al. present an alternative hypothesis to that of Ricci-Vitiani et al. by showing that GSCs can give rise to vascular pericytes (that may actively remodel perivascular niches) which also express Tie2, rather than endothelial cells. Targeting these GSC-derived pericytes disrupted vessel function and inhibited tumor size similarly as the results presented by Ricci-Vitiani et al. for targeting endothelial cells [16]. El Hallani et al. suggested that rather than transdifferentiating, the GSCs were fusing with endothelial cells to create a hybrid tumor vasculature $[139,140]$. Conversely, using a GSC mouse xenograft, Lathia et al. did not mention the integration of tumor-derived cells into the vascular wall [8]. 
Zheng et al. found that, unlike circulating EPCs, the endothelial progenitor cells (EPCs) present in the tumor tissues share genetic aberrations with the tumor cells. The presence of genetic aberrations of glioma cells (EGFR amplification, PTEN deletion, and aneusomy of chromosomes 7 and 10) in intratumoral EPCs may point to transdifferentiation of GSCs into EPCs [141].

It has recently been suggested that GSCs localized near perivascular niches promote angiogenesis in GBM, possibly through differentiation into ECs. Alternatively, GSCs can undergo mesenchymal differentiation and may differentiate into tumor pericytes [142]. Cheng et al. showed that most pericytes were derived from neoplastic cells in human GBM specimens by combined analyses of common genetic changes and the expression of pericyte marker including $\alpha$ smooth muscle actin ( $\alpha$-SMA), NG2, and platelet-derived growth factor receptor (PDGFR), in vitro [16, 17]. By means of lineage-tracing analysis in vivo, they showed that GSCs gave rise to the majority of vascular pericytes in GBM xenografts but did not generate tumor ECs. These GSCderived cells expressed a panel of pericyte markers; however, they no longer expressed putative GSC markers, indicative of commitment to the pericyte lineage. Pericytes isolated from primary human GBMs or xenografts harbored the same genetic alterations as matched GSCs, suggesting that vascular pericytes predominantly derive from neoplastic cells. TGF- $\beta$ signaling from ECs induced differentiation of GSCs into pericytes, at least in part; it was dependent on recruitment by EC-secreted stromal cell-derived factor 1 (SDF-1), which signaled through CXCR4 expressed on GSCs. Selective elimination/deletion of GSC-derived pericytes in tumor-bearing mice disrupted tumor vessel structure and impaired vascular function, resulting in inhibition of tumor growth and prolonged survival. Based on these results, GSCmediated remodeling of the perivascular niche enables GBM progression. The results also suggest that targeting these GSC-derived vascular pericytes may suppress tumor growth and limit resistance to current antiangiogenic therapies [17]. A reasonable assumption is that CSC-derived pericytes might be in a "transitional state" during CSC differentiation into ECs, but further investigation is required in order to confirm whether there is a relationship between tumor-derived pericytes and ECs [142].

To sum up, there is a bidirectional cross talk between GSCs and perivascular niche: on one hand, perivascular niches enhance stem-like proprieties of GSCs, promote invasion and metastasis of these cells, and promote GSCs escape from therapy. On the other hand, GSCs promote EC migration and angiogenesis and are involved in the recruitment process of endothelial progenitor cells. However, GSCs induce the remodeling of perivascular niches, generating ECs and pericytes and inducing angiogenesis/vasculogenesis. Elucidation of these vascular processes will offer new mechanistic insights into the malignancy of glioblastomas that are commonly characterized by tumor angiogenesis. These findings highlight the complexity of the cellular constituents of glioma neovascularization which should be taken into account in new antiangiogenic strategies for gliomas.
3.2. The Hypoxic/Perinecrotic Niche. As a diagnostic hallmark of GBM, hypoxia represents an essential aspect of the glioma microenvironment. Hypoxia promotes tumor angiogenesis, cancer aggression, and therapeutic resistance to various therapies [4]. It also supports GSC self-renewal, proliferation, and tumorigenicity and can induce non-GSCs to acquire GSC features and increased tumorigenic potential [143]. Hypoxia stimulates the expression of the transcription factor, hypoxia-inducible factor (HIF) family. This results in the production of proangiogenic growth factors [107]. Thus, several current publications suggest that the hypoxic niche has a pivotal role in the maintenance and expansion of GSCs [58].

Mediated HIF-1 and HIF-2 represent mediated responses to hypoxia. They comprise a $\beta$ subunit (oxygen-insensitive) and $\alpha$ subunit (oxygen-regulated) [144]. Remarkably, HIF$2 \alpha$ is particularly involved in the activation of signaling pathways regulating stem cell maintenance [145]. HIF- $2 \alpha$ is still elevated under chronic hypoxia, whereas HIF-1 $\alpha$ only gets transiently upregulated [58].

$\mathrm{Li}$ et al. were the first to report the involvement of the HIF pathway in GSCs [10]. Using xenograft glioma-initiating, in vitro neurosphere formation assays and CD133 expression, they observed significant enhancement of stem cell activity under a hypoxic environment. When either HIF1 $\alpha$ or HIF $2 \alpha$ is silenced by shRNA, stem cell activity under both normoxic and hypoxic environments is reduced. Considering HIF $2 \alpha$ mRNA levels correlate with glioma activity, progression, and prognosis, they highlighted that HIF $2 \alpha$ is crucial for glioma stem cell activity. Since HIF-1 $\alpha$ protein levels may be regulated by posttranscriptional mechanisms, this can result in the lack of correlation between HIFl $\alpha$ mRNA levels and stem cell activity [146].

GSCs are enriched in perinecrotic regions of human glioblastoma biopsies. They are characterized by reduced oxygen tension and activation of HIF- $1 \alpha$ and HIF- $2 \alpha$ [147]. In culture, hypoxia upregulates HIF- $1 \alpha$ and HIF- $2 \alpha$ in GSCs. HIF- $2 \alpha$ is directly involved in promoting the GSC phenotype, whereas HIF- $1 \alpha$ appears to play a more general, permissive role in GSC maintenance, possibly by enabling cell survival. Furthermore, HIF- $1 \alpha$ is expressed in both GSCs and nonGSC cells, whereas HIF- $2 \alpha$ is specifically expressed in GSCs $[10,147]$. HIF- $2 \alpha$ upregulates key genes involved in the induction of a pluripotent state [148], including Klf4 and the direct HIF-2 targets Sox 2 and Oct4 $[149,150]$. Besides, HIF- $2 \alpha$ activates c-Myc, another fundamental stem cell regulator, by promoting its interaction with the transcriptional cofactors Spl, Mizl, and Max [151], suggesting that HIF- $2 \alpha$ is a key regulator of the undifferentiated phenotype of GSCs in the hypoxic niche.

3.2.1. Soluble Factors. VEGF expression in both GSCs and non-GSCs is induced by hypoxic conditions, but the VEGF levels are constantly higher in GSCs [10]. High-level production of VEGF by GSCs can promote angiogenesis and their tumor-initiating capacity [19]. The upregulation of VEGF signaling as well as promotion of angiogenesis is highly influenced by HIF, resulting in maintenance of the tumor and its microenvironment [152]. 


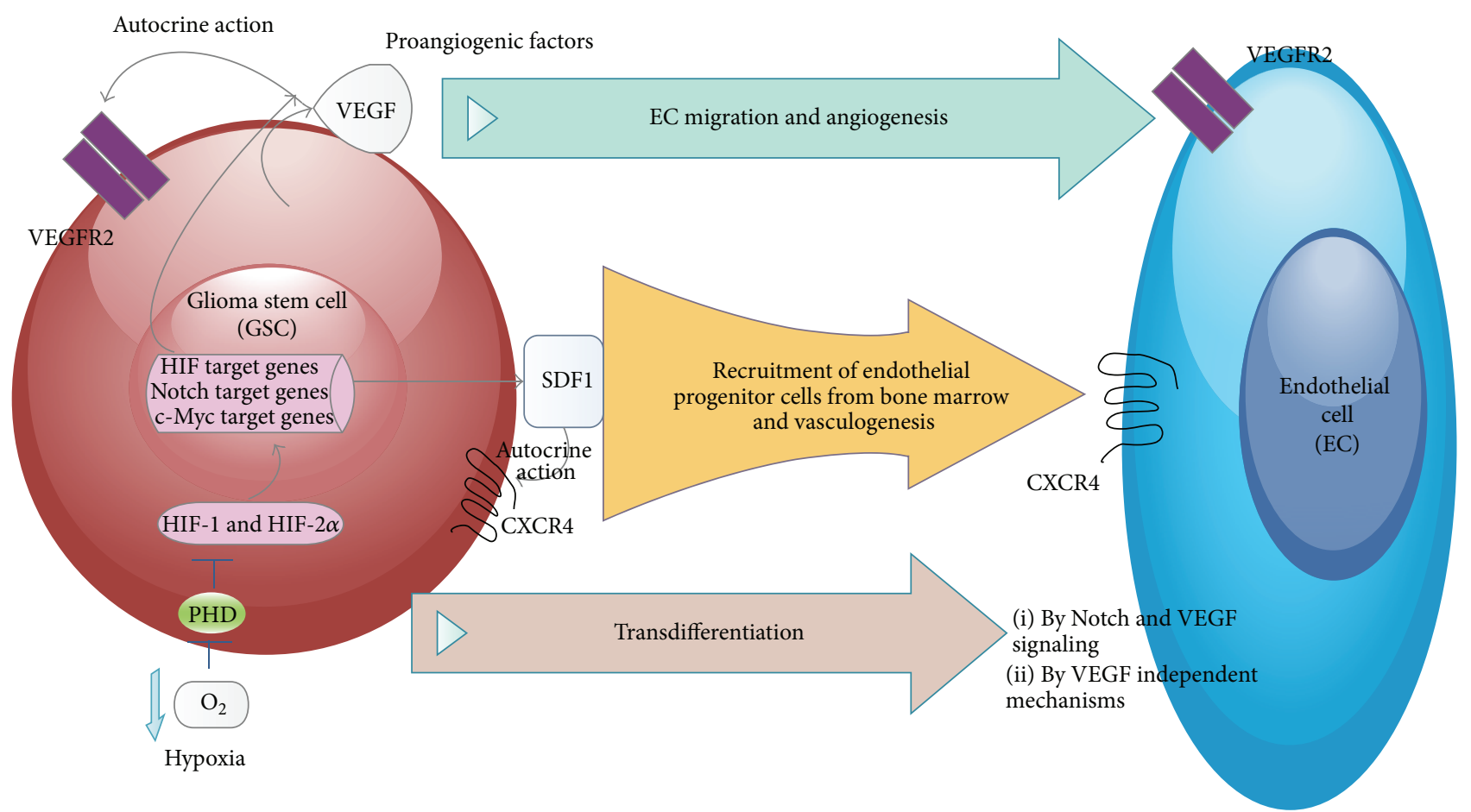

FIGURE 2: Effects of hypoxia on GSCs and effects of GSCs on the endothelial cells. GSCs produce proangiogenic growth factors VEGF and $H D G F$ that stimulate EC migration and angiogenesis; SDF-1 stimulates recruitment of endothelial progenitor cells from bone marrow and vasculogenesis; GSCs can transdifferentiate under hypoxic condition into ECs.

Recently, evidence has emerged indicating that antiangiogenic therapies may induce a more invasive phenotype in recurrent tumors $[153,154]$. A more hypoxic microenvironment induced by vessel regression is believed to be the important cause of a switch to a more invasive program [155]. In addition, hypoxia leads to enrichment of GSCs, with a more invasive phenotype. Therefore, when exploring new antiangiogenic strategy, how to prune excessive vessels without aggravating hypoxia should be taken into consideration.

Recruitment of endothelial and pericyte progenitor cells to promote neovascularization in glioblastoma and regulate the invasion of GBM cells could be induced by HIF1 $\alpha$, partly through increases in SDFl $\alpha$ [156] (Figure 2).

3.2.2. Cells: Cell to Cell Communication. Since phosphatidylinositol 3-kinase (PI3K)/Akt and ERK1/2 pathways inhibition reduced the fraction of CD133+ GSCs, hypoxia-driven GSC expansion depends on them [157]. Under hypoxic conditions, the Notch pathway is also activated in GSCs [158], through HIF- $1 \alpha$. Notch activation led to upregulating the expression of GSC markers such as CD133, Nestin, Bmil, and Olig2, maintaining GSCs pool and phenotype, and growth of tumor neurospheres and xenografts [147]. Notch inhibition resulted in reduced proliferation and increased apoptosis of GSCs, associated with decreased Akt and STAT3 phosphorylation [159]. HIF-1 $\alpha$ induced activation of Notch pathway is critical for hypoxia-mediated maintenance of GSC. Either depletion of HIF- $1 \alpha$ or inactivation of Notch signaling partly inhibits the hypoxia-mediated maintenance of GSCs [160].
Hypoxia upregulates various additional genes involved in the regulation of GSC, such as CXCR4 [157], lysyl oxidase (LOX), hypoxia inducible gene 2 (HIG2) [158], HIF-2 target genes glucose transporter 1 (GLUT1), the proteinase inhibitor Serpin B9, Oct4, and VEGF [10].

GSCs expressed higher levels of histone methyltransferase mixed-lineage leukemia 1 (MLL1) induced by hypoxia than matched nonstem tumor cells, and depletion of MLL1 inhibited HIF transcripts and then reduced the self-renewal, growth, and tumorigenicity of GSCs [161].

When compared to tumors without a mutation, HIF$1 \alpha$ levels were higher in human gliomas harboring an IDH1 mutation. Hence, IDH1 seems to work as a tumor suppressor that, when mutationally inactivated, participates to tumorigenesis, partly through induction of the HIF-1 pathway [162].

3.2.3. Metabolic Reprogramming. Hypoxia is also responsible for metabolic reprogramming, leading to acidification of the tumor microenvironment. Acidic conditions promote the expression of GSC markers, self-renewal, and tumor growth in gliomas. GSCs exert paracrine effects on tumor growth through elaboration of angiogenic factors, and low $\mathrm{pH}$ conditions increase this expression associated with induction of HIF $2 \alpha$. The induction of HIF $2 \alpha$ and other GSC markers by acidic stress can be reverted by elevating $\mathrm{pH}$ in vitro, suggesting that raising intratumoral $\mathrm{pH}$ may be beneficial for targeting the GSC phenotype. Therefore, when exposing to low $\mathrm{pH}$, it promotes malignancy through the induction of a GSCs phenotype, and culturing cancer cells at lower $\mathrm{pH}$ 
reflective of endogenous tumor conditions may better retain the cellular heterogeneity found in tumors $[163,164]$.

3.2.4. miRNAs. miRNAs act as critical mediators of hypoxia signaling according to recent studies reported [165]. The pioneering work of Ivan's team demonstrates that a specific set of hypoxia-regulated miRNAs (HRMs) modulates cell cycle, apoptosis, and DNA repair pathways in response to hypoxia in breast cancer [166, 167]. miR-210-3p has been found highly induced in hypoxic glioma cell lines (U87MG and U251MG) and in hypoxic GBM tumor samples, pointing to its use as a hypoxia marker or therapeutic target in GBM. Several studies have since then found that HRMs fine-tune their hypoxic response through cellular mechanisms, such as angiogenesis, cell cycle regulation, metabolism, apoptosis, metastasis, proliferation, and resistance to anticancer therapy $[168,169]$. GBM survival in the tumor microenvironment is promoted by miR-210-3p, along with aggressiveness by imparting temozolomide resistance and targets HIF3A, which is known to function as a negative regulator of hypoxia-inducible gene expression [170]. Agrawal et al. point to miR-210-3p as an oncogenic player and a novel potential intrinsic marker of hypoxia in glioblastoma [171].

In conclusion, a crucial regulatory role for the GSC phenotype is played by the hypoxic microenvironment, by directly inducing the expression of self-renewal genes, suppressing differentiation, and promoting the cross talk between HIFs and other signaling pathways required for GSC maintenance.

These discoveries emphasize the key role of the microenvironment in regulating the differentiation status of tumor cells and its possible involvement in controlling the plasticity of the cancer stem cell hierarchy.

3.3. The Immune Niche. The immune system appears to have a central role in the control of tumor progression [172]. Recent studies show a direct interaction of GSCs with immune cells, highlighting the major role of these components in the GSCs niche. Furthermore, GSCs and inflammatory cells are involved in a dynamic cross talk involving GSC-mediated induction of immune cell infiltration, generation of a protumorigenic inflammatory environment, and inflammationdriven cancer promotion [58].

Tumor-associated macrophages (TAMs) represent prevalent tumor-infiltrating inflammatory cells in GBM $[173,174]$. The great number of TAMs in GSCs niche suggests their key role in GBM tumor progression, also positively correlated with the malignancy grade [175]. TAMs are mainly located near CD133+ GSCs, around microvessels [176] and in hypoxic areas [177], suggesting a direct interaction between GSCs and TAMs. Enhanced expression of proinflammatory genes like RAGE, COX2, and NF- $\kappa \mathrm{B}$ was recently found in hypoxic niche of GSCs [178]. When compared to differentiated tumor cells, the GSCs show an increased capacity in active chemoattraction and recruitment of TAMs, processes mediated by chemokines and growth factors, secreted by GSCs, including VEGF, neurotensin, SDF1, and soluble colony-stimulating factor 1 (sCSF-1) [177, 179]. GSCs also secrete factors that support the growth of macrophages and induce the polarization of TAMs into the immunosuppressive M2 phenotype [68].

Although the above-mentioned molecules prove the important roles of GSCs in immune cell modulation, leading to the induction of tumor promoting inflammation, understanding the impact of immune cells on GSC maintenance is still limited. Molecules/cytokines like TGF $\beta$, VEGF, SDF1, bFGF, and NO produced by immune cells [180-182] have been independently proven to maintain and promote GSCs [58], pointing to speculate that the protumorigenic function of specific sets of inflammatory cells is additionally mediated through the direct stimulation of GSCs, which will most certainly represent a motivating area of research in further studies.

The origin of TAMs, whether they are recruited from locally activated microglia or from the peripheral monocyte population, represents an important question to be answered, with high addressability to potential future therapy targets. Oncogenically, microglia are the only resident macrophages that are known to be exclusively derived from yolk sac macrophages without monocyte intermediates. To maintain their population, they rely on local proliferation [183]. Recent studies have identified a unique microglial subpopulation as an indispensable component of the subventricular neurogenetic zone and rostral migratory stream, establishing the framework for a preexisting collaborative cross talk between brain resident immune and stem cells [184].

Over two decades ago, the relationship between microglia and brain tumors has been first mentioned [175, 185], when they were identified as the "Achilles heel" of the immune system [186], exerting a surprisingly low cytotoxic activity. In vitro long-term cocultures of glioma and microglial cells showed a short activation of phagocytic properties, followed by a steady state depression.

In 2015, Zhou et al. provide new insights into where and how TAMs are recruited and educated by GSCs in GBMs [187]. They have analyzed the molecular relationship between GSCs and TAM recruitment in GBMs and demonstrated that GSCs secrete periostin (POSTN), a GSCsecreted cytokine, to potently attract and recruit peripheral monocytes. The correlation between TAM density and glioma grade points to a supportive role for TAMs in tumor progression, which can be altered by POSTN-directed blockade.

Inhibition of macrophage-colony stimulation factor receptor demonstrated myeloid compartment involvement in glioma initiation and progression [188]. The penetration of vascular borne cells into tumoral tissue offers vehicle for peripheral delivery of cytotoxic drugs, in an otherwise protected environment. Apart from blood brain barrier diffusibility, therapeutic monocytes have the advantage of tissue depth diffusion, almost three times higher than nanoparticles [189].

Accumulated knowledge on TAMs and GSCs roles on immune cell modulation, possibly delineating a third niche, underlined the role of immune system in GBM progression and GSCs escape from therapy. 


\section{Challenges in Targeting the Tumor Microenvironment}

Cancer tissue does not represent a homogenous population of clonally expanded cancer cells; this constitutes an important paradigm shift in cancer research [190]. GSCs have a high capacity for self-renewal and tumorigenic potential [191].

Since conventional therapeutic approaches have not been developed to target GSCs, many such cells are enriched by conventional cancer therapy [35]. The unsuccessful removal of GSCs constitutes an important reason for which cancer relapse follows conventional therapy and, thus, it is a major obstacle to efficient cancer treatment [192]. Cancer cells and host cells form a tumor microenvironment that allows tumor initiation and progression. Because conventional cancer therapy approaches have been developed without emphasizing the tumor microenvironment, a key new focus for cancer therapy is to limit cancer development by targeting GSCs microenvironment: perivascular niche, hypoxic niche, and immune evasion [193].

Interrupting the perivascular niche might prove a critical approach for GSCs targeting. Directly targeting endothelial cells that constitute the tumor vasculature is an alternative approach that has been used to destabilize GSCs function. Thus, a modification of this microenvironment can decrease GSCs tumorigenicity. One approach is the use of antiangiogenic drugs, which decrease blood flow toward the tumor and induce local alterations to finally decrease the number of GSCs or render them sensitive to other therapies [194]. The monoclonal antibody bevacizumab (Avastin) and the novel small molecule pan-VEGF inhibitor cediranib (AZD2171) that targets VEGF (in order to disrupt VEGF/VEGFR interaction) are currently used in clinical therapy somewhat successfully. Treating mice bearing GSCs initiated xenografts with bevacizumab or other antiangiogenic agents (e.g., antiSDF1 drug AMD3100) delayed tumor growth in vivo due in part to a decrease in tumor blood vessels as well as the percentage of GSCs [195]. Considering the use of antiangiogenic drugs has become widespread, it has been found that tumors develop mechanisms of resistance to antiangiogenic drugs. Recent studies have raised concerns that system antiVEGF treatment may improve short-term patient outcome but may ultimately lead to more aggressive malignancies. By "pruning" leaky vessels, anti-VEGF drugs like bevacizumab may in fact improve overall tumor vasculature. This could lead to increased invasion and more aggressive growth [196]. Combining antiangiogenic treatment with cytotoxic agents leads to xenografted glioma tumors depleted of GSCs and reduced in size after treatment [197].

IL-6 is another secreted factor known to support angiogenesis, which can be produced by the tumor microenvironment. Higher levels of IL- 6 mRNA are directly linked to poor patient survival in GBM. IL-6 receptors, gp130 and IL-6R $\alpha$, are preferentially expressed on GSCs and their expression can be stimulated by hypoxia, important component of the tumor microenvironment. Directly targeting IL-6 or IL-6R $\alpha$ by shRNA impairs GSC growth and survival in vitro, suggesting the significance of IL- 6 autocrine signals in GSC maintenance [50]. Notably, administration of anti-IL-6 antibody delayed the growth of tumors initiated with GSCs, suggesting that targeting IL-6 may be useful as antiglioma therapies [196].

Angiogenesis, cell migration, and tumor resistance are induced by hypoxic microenvironment. Therefore, HIFs constitute an important molecular target to be developed for novel therapeutic strategies in order to inhibit GBM malignant progression [198]. An increasing number of chemical compounds have been shown to inhibit HIF activity through a wide variety of molecular processes and to counteract tumor growth in GBM xenograft models. There are several molecular effects driven by these compounds, such as decreased levels of HIF- $1 \alpha$, mRNA, and protein synthesis, inhibition of HIF- $\alpha$ heterodimerization with ARNT, block of HIFs binding to DNA and decrease of its transcriptional activity, and increased HIF- $1 \alpha$ degradation. Even though the number of inhibitor molecules of HIF-1 has rapidly increased lately, only few compounds are progressing towards preclinical and early clinical development. Remarkably, the combination of HIF-1 inhibitors with existing treatments or new-targeted therapies could prove useful in the clinical practice [199].

It appears that inflammation may be influenced by HIF, including the adaptive and innate inflammatory responses [200-202]. The shared requirement for HIF in GSCs and inflammatory cells raised the interesting prospect that GSCs and inflammation, two important challenges in cancer therapy, may be addressed by targeting HIF [146].

The hypoxic niche and HIF-1 $\alpha$ have been reported to enhance the migration properties of GSCs by promoting metalloproteinase expression and migration-associated receptors, such as CXCR4 [203]. These data are supported by the hypothesis that most hypoxic cells could migrate through the above-described layers [204], potentially invade normal brain tissues, or maintain the GSC population of the most peripheral layers. For this reason, the migratory features of GBM cells could prove to be a valid therapeutic target for this tumor.

The key chemokine that has been associated with the migratory process of GBM is the SDF-1/CXCL12. The activated CXCR4/CXCL12 complex is rapidly internalized from the cell surface. GBM cells are endowed with a high expression of CXCR4. Moreover, a strict correlation between CXCR4 levels and the infiltrative extension of GBM tumors exists. Recently, it has been postulated that this receptor might be a cell surface marker for GSCs [129]. Another process by which the CXCR4/CXCL12 axis contributes to GBM growth is its ability to recruit endothelial and marrow cells to support tumor vasculogenesis and angiogenesis [205]. In this context, plerixafor (AMD3100) is a bicyclam molecule that antagonizes the binding of SDF-1 to CXCR4 and inhibits irradiation-induced vasculogenesis in vivo [195].

Hypoxic stimuli are not the same all over tumor mass and different zones described for each GBM have been mentioned recently. Genome, transcriptome, and methylome analysis of different areas of the same tumor (necrotic zone, tumor zone, interface, and peripheral brain zone) revealed that "transcriptome heterogeneity was much more important within tumors than between patients." Tumor subtype, as assessed by 840 gene signatures, differed between the aforementioned zones: the neural and proneural subtypes 
were located in peripheral brain zone and interface, whereas mesenchymal and classical subtypes were found in tumor and necrotic zones [206]. These results would imply that aggressive subtypes are peripherally located, favoring local invasion into healthy tissue. This brings into focus a new microenvironment, the peripheral brain zone, which harbors $90 \%$ of tumor recurrences [207]. This area includes, apart from infiltrated aggressive tumor cells, reactive astrocytes, inflammatory cells, and "glioblastoma-associated stromal cells." These stromal cells are diploid, share phenotypic and functional properties with cancer-associated fibroblasts, and do not recapitulate the genomic alterations typical of tumor cells [208].

Immune cell recruiting into tumoral zone was viewed as a "Trojan horse" for cytotoxic drug delivery. The major problem to overcome was the cytotoxicity of the load that would affect the very cargo that carries it. The alternative to overcome this problem is loading with nanoparticles for photothermal therapy [209] or increase delivery through opening the blood brain barrier [210].

To conclude, the discoveries of all these studies suggest that both the hypoxic and the perivascular niche could prove an efficient target for GSCs treatment. Understanding the biological behavior of GSCs, their regulatory processes and their niches may directly impact current efforts for directed therapeutics against the highly aggressive gliomas [152]. Consequently, multimodal therapies that include combinations of antiangiogenic therapies along with cytotoxic therapies should be able to overcome this problem. The simple eradication of the existing GSCs is not enough to provide a cure for gliomas; however, obstructing the potential sources of GSCs as well as ameliorating the local tumor inducing/promoting microenvironment represents a reasonable strategy [211].

\section{Conclusions}

There is an urgent need for understanding the cross talk between GSCs and their niches, which supports the GSCs self-renewal, tumor invasion, and metastasis, as well as GSCs escape from therapy. Although many questions and controversies remain, the progress has been driven by the interest in the microenvironment that induces particular signatures in order to regulate GSCs maintenance and function. Novel therapeutical approaches should disrupt the protective niches, perivascular, hypoxic, and immune, of GSCs, in order to improve and even to revolutionize current diagnosis and therapy of gliomas. Therefore, effective control of the GSCs microenvironment will likely complement the conventional approach of cancer therapy, aiming at eradicating GSCs.

\section{Conflict of Interests}

The authors declare that they have no conflict of interests.

\section{Authors' Contribution}

The authors contributed equally to this work. All authors read and approved the final paper.

\section{Acknowledgments}

This paper is partly supported by the Sectorial Operational Programme Human Resources Development (SOPHRD), financed by the European Social Fund and the Romanian Government under Contract number POSDRU 141531. This work was partly supported by a grant of the Romanian National Authority for Scientific Research and Innovation, CNCS-UEFISCDI, Project numbers PN-II-RU-TE2014-4-1879, PN-II-RU-TE-2014-4-1534, 101/01.10.2015, PNII 265/2014, PNII 93/2012, and PNII 192/2014. The authors would like to thank Irina Radu, certified translator in medicine and pharmacy (certificate credentials: series: E no.: 0048), for professional linguistic assistance.

\section{References}

[1] R. Stupp, M. E. Hegi, W. P. Mason et al., "Effects of radiotherapy with concomitant and adjuvant temozolomide versus radiotherapy alone on survival in glioblastoma in a randomised phase III study: 5-year analysis of the EORTC-NCIC trial," The Lancet Oncology, vol. 10, no. 5, pp. 459-466, 2009.

[2] M. E. Hardee and D. Zagzag, "Mechanisms of glioma-associated neovascularization," The American Journal of Pathology, vol. 181, no. 4, pp. 1126-1141, 2012.

[3] A. L. Vescovi, R. Galli, and B. A. Reynolds, "Brain tumour stem cells," Nature Reviews Cancer, vol. 6, no. 6, pp. 425-436, 2006.

[4] D. L. Schonberg, D. Lubelski, T. E. Miller et al., "Brain tumor stem cells: molecular characteristics and their impact on therapy," Molecular Aspects of Medicine, vol. 39, pp. 82-101, 2014.

[5] R. Galli, E. Binda, U. Orfanelli et al., "Isolation and characterization of tumorigenic, stem-like neural precursors from human glioblastoma," Cancer Research, vol. 64, no. 19, pp. 7011-7021, 2004.

[6] D. Beier, B. Schriefer, K. Brawanski et al., "Efficacy of clinically relevant temozolomide dosing schemes in glioblastoma cancer stem cell lines," Journal of Neuro-Oncology, vol. 109, no. 1, pp. 45-52, 2012.

[7] J. Chen, Y. Li, T.-S. Yu et al., "A restricted cell population propagates glioblastoma growth after chemotherapy," Nature, vol. 488, no. 7412, pp. 522-526, 2012.

[8] J. D. Lathia, J. Gallagher, J. T. Myers et al., "Direct in vivo evidence for tumor propagation by glioblastoma cancer stem cells," PLoS ONE, vol. 6, no. 9, Article ID e24807, 2011.

[9] C. Calabrese, H. Poppleton, M. Kocak et al., "A perivascular niche for brain tumor stem cells," Cancer Cell, vol. 11, no. 1, pp. 69-82, 2007.

[10] Z. Li, S. Bao, Q. Wu et al., "Hypoxia-inducible factors regulate tumorigenic capacity of glioma stem cells," Cancer Cell, vol. 15, no. 6, pp. 501-513, 2009.

[11] T. S. Zhu, M. A. Costello, C. E. Talsma et al., "Endothelial cells create a stem cell niche in glioblastoma by providing NOTCH ligands that nurture self-renewal of cancer stem-like cells," Cancer Research, vol. 71, no. 18, pp. 6061-6072, 2011.

[12] D. W. Infanger, Y. Cho, B. S. Lopez et al., "Glioblastoma stem cells are regulated by interleukin- 8 signaling in a tumoral perivascular niche," Cancer Research, vol. 73, no. 23, pp. 70797089, 2013. 
[13] E. M. Galan-Moya, A. Le Guelte, E. L. Fernandes et al., "Secreted factors from brain endothelial cells maintain glioblastoma stem-like cell expansion through the mTOR pathway," EMBO Reports, vol. 12, no. 5, pp. 470-476, 2011.

[14] R. Wang, K. Chadalavada, J. Wilshire et al., "Glioblastoma stemlike cells give rise to tumour endothelium," Nature, vol. 468, no. 7325, pp. 829-833, 2010.

[15] L. Ricci-Vitiani, R. Pallini, M. Biffoni et al., "Tumour vascularization via endothelial differentiation of glioblastoma stem-like cells," Nature, vol. 468, no. 7325, pp. 824-830, 2010.

[16] L. Cheng, Z. Huang, W. Zhou et al., "Glioblastoma stem cells generate vascular pericytes to support vessel function and tumor growth," Cell, vol. 153, no. 1, pp. 139-152, 2013.

[17] P. O. Guichet, S. Guelfi, M. Teigell et al., "Notch1 stimulation induces a vascularization switch with pericyte-like cell differentiation of glioblastoma stem cells," Stem Cells, vol. 33, no. 1, pp. 21-34, 2015.

[18] D. Chroscinski, D. Sampey, and N. Maherali, "Registered report: tumour vascularization via endothelial differentiation of glioblastoma stem-like cells," eLife, vol. 4, 2015.

[19] S. Bao, Q. Wu, S. Sathornsumetee et al., "Stem cell-like glioma cells promote tumor angiogenesis through vascular endothelial growth factor," Cancer Research, vol. 66, no. 16, pp. 7843-7848, 2006.

[20] C. Folkins, Y. Shaked, S. Man et al., "Glioma tumor stem-like cells promote tumor angiogenesis and vasculogenesis via vascular endothelial growth factor and stromal-derived factor 1," Cancer Research, vol. 69, no. 18, pp. 7243-7251, 2009.

[21] C. Thirant, E.-M. Galan-Moya, L. G. Dubois et al., "Differential proteomic analysis of human glioblastoma and neural stem cells reveals HDGF as a novel angiogenic secreted factor," STEM CELLS, vol. 30, no. 5, pp. 845-853, 2012.

[22] S. K. Singh, I. D. Clarke, T. Hide, and P. B. Dirks, "Cancer stem cells in nervous system tumors," Oncogene, vol. 23, no. 43, pp. 7267-7273, 2004.

[23] R. M. R. Gangemi, F. Griffero, D. Marubbi et al., "SOX2 silencing in glioblastoma tumor-initiating cells causes stop of proliferation and loss of tumorigenicity," STEM CELLS, vol. 27, no. 1, pp. 40-48, 2009.

[24] J. Anido, A. Sáez-Borderías, A. Gonzàlez-Juncà et al., “TGF- $\beta$ receptor inhibitors target the $\mathrm{CD} 44^{\text {high }} / \mathrm{Id} 11^{\text {high }}$ glioma-initiating cell population in human glioblastoma," Cancer Cell, vol. 18, no. 6, pp. 655-668, 2010.

[25] J. D. Lathia, J. Gallagher, J. M. Heddleston et al., "Integrin alpha 6 regulates glioblastoma stem cells," Cell Stem Cell, vol. 6, no. 5, pp. 421-432, 2010.

[26] N. Thon, K. Damianoff, J. Hegermann et al., "Presence of pluripotent $\mathrm{CD}_{133^{+}}$cells correlates with malignancy of gliomas," Molecular and Cellular Neuroscience, vol. 43, no. 1, pp. 51-59, 2010.

[27] D. Bexell, S. Gunnarsson, P. Siesjö, J. Bengzon, and A. Darabi, "CD133+ and nestin+ tumor-initiating cells dominate in N29 and N32 experimental gliomas," International Journal of Cancer, vol. 125, no. 1, pp. 15-22, 2009.

[28] M. Zhang, T. Song, L. Yang et al., "Nestin and CD133: valuable stem cell-specific markers for determining clinical outcome of glioma patients," Journal of Experimental and Clinical Cancer Research, vol. 27, no. 1, article 85, 2008.

[29] Y. Guo, S. Liu, P. Wang et al., "Expression profile of embryonic stem cell-associated genes Oct4, Sox2 and Nanog in human gliomas," Histopathology, vol. 59, no. 4, pp. 763-775, 2011.
[30] J. Mathieu, Z. Zhang, W. Zhou et al., "HIF induces human embryonic stem cell markers in cancer cells," Cancer Research, vol. 71, no. 13, pp. 4640-4652, 2011.

[31] C.-S. Niu, D.-X. Li, Y.-H. Liu, X.-M. Fu, S.-F. Tang, and J. Li, "Expression of NANOG in human gliomas and its relationship with undifferentiated glioma cells," Oncology Reports, vol. 26, no. 3, pp. 593-601, 2011.

[32] H. Ikushima, T. Todo, Y. Ino et al., "Glioma-initiating cells retain their tumorigenicity through integration of the Sox axis and Oct4 protein," Journal of Biological Chemistry, vol. 286, no. 48, pp. 41434-41441, 2011.

[33] Y. Ge, F. Zhou, H. Chen et al., "Sox2 is translationally activated by eukaryotic initiation factor $4 \mathrm{E}$ in human glioma-initiating cells," Biochemical and Biophysical Research Communications, vol. 397, no. 4, pp. 711-717, 2010.

[34] D. Hägerstrand, X. He, M. Bradic Lindh et al., "Identification of a SOX2-dependent subset of tumor- and sphere-forming glioblastoma cells with a distinct tyrosine kinase inhibitor sensitivity profile," Neuro-Oncology, vol. 13, no. 11, pp. 1178-1191, 2011.

[35] S. Bao, Q. Wu, R. E. McLendon et al., "Glioma stem cells promote radioresistance by preferential activation of the DNA damage response," Nature, vol. 444, no. 7120, pp. 756-760, 2006.

[36] G. Liu, X. Yuan, Z. Zeng et al., "Analysis of gene expression and chemoresistance of $\mathrm{CD} 133^{+}$cancer stem cells in glioblastoma," Molecular Cancer, vol. 5, article 67, 2006.

[37] S. C. Li, L. T. Vu, H. W. Ho et al., "Cancer stem cells from a rare form of glioblastoma multiforme involving the neurogenic ventricular wall," Cancer Cell International, vol. 12, no. 1, article 41, 2012.

[38] Q. Huang, Q.-B. Zhang, J. Dong et al., "Glioma stem cells are more aggressive in recurrent tumors with malignant progression than in the primary tumor, and both can be maintained long-term in vitro," BMC Cancer, vol. 8, article 304, 2008.

[39] P. Ø. Sakariassen, H. Immervoll, and M. Chekenya, "Cancer stem cells as mediators of treatment resistance in brain tumors: status and controversies," Neoplasia, vol. 9, no. 11, pp. 882-892, 2007.

[40] J. Wang, T. P. Wakeman, J. D. Lathia et al., "Notch promotes radioresistance of glioma stem cells," STEM CELLS, vol. 28, no. 1, pp. 17-28, 2010.

[41] S. K. Singh, I. D. Clarke, M. Terasaki et al., "Identification of a cancer stem cell in human brain tumors," Cancer Research, vol. 63, no. 18, pp. 5821-5828, 2003.

[42] S. K. Singh, C. Hawkins, I. D. Clarke et al., "Identification of human brain tumour initiating cells," Nature, vol. 432, no. 7015, pp. 396-401, 2004.

[43] K.-I. Mizutani, K. Yoon, L. Dang, A. Tokunaga, and N. Gaiano, "Differential Notch signalling distinguishes neural stem cells from intermediate progenitors," Nature, vol. 449, no. 7160, pp. 351-355, 2007.

[44] J. S. Tchorz, M. Tome, D. Cloëtta et al., "Constitutive Notch2 signaling in neural stem cells promotes tumorigenic features and astroglial lineage entry," Cell Death and Disease, vol. 3, article e325, 2012.

[45] C.-H. Yoon, M.-J. Kim, R.-K. Kim et al., "c-Jun N-terminal kinase has a pivotal role in the maintenance of self-renewal and tumorigenicity in glioma stem-like cells," Oncogene, vol. 31, no. 44, pp. 4655-4666, 2012. 
[46] V. Clement, P. Sanchez, N. de Tribolet, I. Radovanovic, and A. Ruiz i Altaba, "HEDGEHOG-GLI1 signaling regulates human glioma growth, cancer stem cell self-renewal, and tumorigenicity," Current Biology, vol. 17, no. 2, pp. 165-172, 2007.

[47] M. Ehtesham, K. Y. Mapara, C. B. Stevenson, and R. C. Thompson, "CXCR4 mediates the proliferation of glioblastoma progenitor cells," Cancer Letters, vol. 274, no. 2, pp. 305-312, 2009.

[48] R. Bonavia, M. M. Inda, S. Vandenberg et al., "EGFRvIII promotes glioma angiogenesis and growth through the NF-kappaB, interleukin-8 pathway," Oncogene, vol. 31, no. 36, pp. 40544066, 2012.

[49] X. Jin, J. Yin, S.-H. Kim et al., "EGFR-AKT-Smad signaling promotes formation of glioma stem-like cells and tumor angiogenesis by ID3-driven cytokine induction," Cancer Research, vol. 71, no. 22, pp. 7125-7134, 2011.

[50] H. Wang, J. D. Lathia, Q. Wu et al., "Targeting interleukin 6 signaling suppresses glioma stem cell survival and tumor growth," STEM CELLS, vol. 27, no. 10, pp. 2393-2404, 2009.

[51] E. L. Jackson, J. M. Garcia-Verdugo, S. Gil-Perotin et al., "PDGFR $\alpha$-positive B cells are neural stem cells in the adult SVZ that form glioma-like growths in response to increased PDGF signaling," Neuron, vol. 51, no. 2, pp. 187-199, 2006.

[52] J. Lee, M. J. Son, K. Woolard et al., "Epigenetic-mediated dysfunction of the bone morphogenetic protein pathway inhibits differentiation of glioblastoma-initiating cells," Cancer Cell, vol. 13, no. 1, pp. 69-80, 2008.

[53] S. G. M. Piccirillo, B. A. Reynolds, N. Zanetti et al., "Bone morphogenetic proteins inhibit the tumorigenic potential of human brain tumour-initiating cells," Nature, vol. 444, no. 7120, pp. 761-765, 2006.

[54] A. Ruiz i Altaba, C. Mas, and B. Stecca, "The Gli code: an information nexus regulating cell fate, stemness and cancer," Trends in Cell Biology, vol. 17, no. 9, pp. 438-447, 2007.

[55] E. E. Bar, A. Chaudhry, A. Lin et al., "Cyclopamine-mediated hedgehog pathway inhibition depletes stem-like cancer cells in glioblastoma," STEM CELLS, vol. 25, no. 10, pp. 2524-2533, 2007.

[56] Y. Xin, S.-Y. Hao, Y.-J. Tian et al., "Expression and significance of sonic hedgehog signaling pathway-related components in brainstem and supratentorial astrocytomas," Chinese Medical Journal, vol. 124, no. 21, pp. 3515-3520, 2011.

[57] M. L. Cruceru, M. Neagu, J.-B. Demoulin, and S. N. Constantinescu, "Therapy targets in glioblastoma and cancer stem cells: lessons from haematopoietic neoplasms," Journal of Cellular and Molecular Medicine, vol. 17, no. 10, pp. 1218-1235, 2013.

[58] A. Filatova, T. Acker, and B. K. Garvalov, "The cancer stem cell niche(s): the crosstalk between glioma stem cells and their microenvironment," Biochimica et Biophysica Acta-General Subjects, vol. 1830, no. 2, pp. 2496-2508, 2013.

[59] H. Ikushima, T. Todo, Y. Ino, M. Takahashi, K. Miyazawa, and K. Miyazono, "Autocrine TGF- $\beta$ signaling maintains tumorigenicity of glioma-initiating cells through Sry-related HMGbox factors," Cell Stem Cell, vol. 5, no. 5, pp. 504-514, 2009.

[60] R. Schüle, C. Dictus, B. Campos et al., "Potential canonical wnt pathway activation in high-grade astrocytomas," The Scientific World Journal, vol. 2012, Article ID 697313, 11 pages, 2012.

[61] V. Häyry, O. Tynninen, H. K. Haapasalo et al., "Stem cell protein BMI-1 is an independent marker for poor prognosis in oligodendroglial tumours," Neuropathology and Applied Neurobiology, vol. 34, no. 5, pp. 555-563, 2008.
[62] S. Facchino, M. Abdouh, W. Chatoo, and G. Bernier, "BMI1 confers radioresistance to normal and cancerous neural stem cells through recruitment of the DNA damage response machinery," The Journal of Neuroscience, vol. 30, no. 30, pp. 10096-10111, 2010.

[63] M. Zbinden, A. Duquet, A. Lorente-Trigos, S.-N. Ngwabyt, I. Borges, and A. Ruiz I Altaba, "NANOG regulates glioma stem cells and is essential in vivo acting in a cross-functional network with GLI1 and p53," The EMBO Journal, vol. 29, no. 15, pp. 26592674, 2010.

[64] L. Cheng, S. Bao, and J. N. Rich, "Potential therapeutic implications of cancer stem cells in glioblastoma," Biochemical Pharmacology, vol. 80, no. 5, pp. 654-665, 2010.

[65] Y. Yoshida, K. Takahashi, K. Okita, T. Ichisaka, and S. Yamanaka, "Hypoxia enhances the generation of induced pluripotent stem cells," Cell Stem Cell, vol. 5, no. 3, pp. 237-241, 2009.

[66] K. L. Ligon, J. A. Alberta, A. T. Kho et al., “The oligodendroglial lineage marker OLIG2 is universally expressed in diffuse gliomas," Journal of Neuropathology and Experimental Neurology, vol. 63, no. 5, pp. 499-509, 2004.

[67] L. E. Barrett, Z. Granot, C. Coker et al., "Self-renewal does not predict tumor growth potential in mouse models of high-grade glioma," Cancer Cell, vol. 21, no. 1, pp. 11-24, 2012.

[68] A. Wu, J. Wei, L.-Y. Kong et al., "Glioma cancer stem cells induce immunosuppressive macrophages/microglia," Neuro Oncology, vol. 12, no. 11, pp. 1113-1125, 2010.

[69] G.-H. Li, H. Wei, S.-Q. Lv, H. Ji, and D.-L. Wang, "Knockdown of STAT3 expression by RNAi suppresses growth and induces apoptosis and differentiation in glioblastoma stem cells," International Journal of Oncology, vol. 37, no. 1, pp. 103-110, 2010.

[70] M. M. Sherry, A. Reeves, J. K. Wu, and B. H. Cochran, "STAT3 is required for proliferation and maintenance of multipotency in glioblastoma stem cells," STEM CELLS, vol. 27, no. 10, pp. 23832392, 2009.

[71] A. C. deCarvalho, K. Nelson, N. Lemke et al., "Gliosarcoma stem cells undergo glial and mesenchymal differentiation in vivo," STEM CELLS, vol. 28, no. 2, pp. 181-190, 2010.

[72] M. Visani, D. de Biase, G. Marucci et al., "Expression of 19 microRNAs in glioblastoma and comparison with other brain neoplasia of grades I-III," Molecular Oncology, vol. 8, no. 2, pp. 417-430, 2014.

[73] E. V. Makeyev, J. Zhang, M. A. Carrasco, and T. Maniatis, "The microRNA miR-124 promotes neuronal differentiation by triggering brain-specific alternative pre-mRNA splicing," Molecular Cell, vol. 27, no. 3, pp. 435-448, 2007.

[74] Y. Zhang, A. Dutta, and R. Abounader, "The role of microRNAs in glioma initiation and progression," Frontiers in Bioscience, vol. 17, no. 2, pp. 700-712, 2012.

[75] J. Silber, A. Jacobsen, T. Ozawa et al., "miR-34a repression in proneural malignant gliomas upregulates expression of its target PDGFRA and promotes tumorigenesis," PLoS ONE, vol. 7, no. 3, Article ID e33844, 2012.

[76] H. Gal, G. Pandi, A. A. Kanner et al., "MIR-451 and imatinib mesylate inhibit tumor growth of Glioblastoma stem cells," Biochemical and Biophysical Research Communications, vol. 376, no. 1, pp. 86-90, 2008.

[77] M. Smits, J. Nilsson, S. E. Mir et al., "miR-101 is down-regulated in glioblastoma resulting in EZH2-induced proliferation, migration, and angiogenesis," Oncotarget, vol. 1, no. 8, pp. 710$720,2010$. 
[78] Y. Tu, X. Gao, G. Li et al., "MicroRNA-218 inhibits glioma invasion, migration, proliferation, and cancer stem-like cell selfrenewal by targeting the polycomb group gene Bmil," Cancer Research, vol. 73, no. 19, pp. 6046-6055, 2013.

[79] J. Godlewski, M. O. Nowicki, A. Bronisz et al., "MicroRNA451 regulates LKB1/AMPK signaling and allows adaptation to metabolic stress in glioma cells," Molecular Cell, vol. 37, no. 5, pp. 620-632, 2010.

[80] X. Zhou, J. Zhang, Q. Jia et al., "Reduction of miR-21 induces glioma cell apoptosis via activating caspase 9 and 3," Oncology Reports, vol. 24, no. 1, pp. 195-201, 2010.

[81] F. Zhi, X. Chen, S. Wang et al., "The use of hsa-miR-21, hsa-miR$181 \mathrm{~b}$ and hsa-miR-106a as prognostic indicators of astrocytoma," European Journal of Cancer, vol. 46, no. 9, pp. 1640-1649, 2010.

[82] L. Sun, W. Yan, Y. Wang et al., "MicroRNA-10b induces glioma cell invasion by modulating MMP-14 and uPAR expression via HOXD10," Brain Research, vol. 1389, pp. 9-18, 2011.

[83] B. Malzkorn, M. Wolter, F. Liesenberg et al., "Identification and functional characterization of microRNAs involved in the malignant progression of gliomas," Brain Pathology, vol. 20, no. 3, pp. 539-550, 2010.

[84] S. A. M. Rao, V. Santosh, and K. Somasundaram, "Genomewide expression profiling identifies deregulated miRNAs in malignant astrocytoma," Modern Pathology, vol. 23, no. 10, pp. 1404-1417, 2010.

[85] N. Wu, L. Xiao, X. Zhao et al., "miR-125b regulates the proliferation of glioblastoma stem cells by targeting E2F2," FEBS Letters, vol. 586, no. 21, pp. 3831-3839, 2012.

[86] L. Shi, Y. Wan, G. Sun et al., "Functional differences of miR$125 \mathrm{~b}$ on the invasion of primary glioblastoma CD133-negative cells and CD133-positive cells," NeuroMolecular Medicine, vol. 14, no. 4, pp. 303-316, 2012.

[87] Y. Guan, M. Mizoguchi, K. Yoshimoto et al., "MiRNA-196 is upregulated in glioblastoma but not in anaplastic astrocytoma and has prognostic significance," Clinical Cancer Research, vol. 16, no. 16, pp. 4289-4297, 2010.

[88] H. Yan, D. W. Parsons, G. Jin et al., "IDH1 and IDH2 mutations in gliomas," The New England Journal of Medicine, vol. 360, no. 8, pp. 765-773, 2009.

[89] H. He, C. S. Niu, and M. W. Li, "Correlation between glioblastoma stem-like cells and tumor vascularization," Oncology Reports, vol. 27, no. 1, pp. 45-50, 2011.

[90] K. B. Pointer, P. A. Clark, M. Zorniak, B. M. Alrfaei, and J. S. Kuo, "Glioblastoma cancer stem cells: biomarker and therapeutic advances," Neurochemistry International, vol. 71, no. 1, pp. 1-7, 2014.

[91] F. Gambelli, F. Sasdelli, I. Manini et al., "Identification of cancer stem cells from human glioblastomas: growth and differentiation capabilities and CD133/prominin-1 expression," Cell Biology International, vol. 36, no. 1, pp. 29-38, 2012.

[92] D. Beier, P. Hau, M. Proescholdt et al., "CD133' ${ }^{+}$and $\mathrm{CD} 133^{-}$ glioblastoma-derived cancer stem cells show differential growth characteristics and molecular profiles," Cancer Research, vol. 67, no. 9, pp. 4010-4015, 2007.

[93] K. M. Joo, S. Y. Kim, X. Jin et al., "Clinical and biological implications of CD133-positive and CD133-negative cells in glioblastomas," Laboratory Investigation, vol. 88, no. 8, pp. 808815, 2008.

[94] T. Yang and K. Rycaj, "Targeted therapy against cancer stem cells," Oncology Letters, vol. 10, no. 1, pp. 27-33, 2015.
[95] R. Chen, M. C. Nishimura, S. M. Bumbaca et al., "A hierarchy of self-renewing tumor-initiating cell types in glioblastoma," Cancer Cell, vol. 17, no. 4, pp. 362-375, 2010.

[96] P. Brescia, B. Ortensi, L. Fornasari, D. Levi, G. Broggi, and G. Pelicci, "CD133 is essential for glioblastoma stem cell maintenance," STEM CELLS, vol. 31, no. 5, pp. 857-869, 2013.

[97] B. Wu, C. Sun, F. Feng, M. Ge, and L. Xia, "Do relevant markers of cancer stem cells CD133 and Nestin indicate a poor prognosis in glioma patients? A systematic review and meta-analysis," Journal of Experimental \& Clinical Cancer Research, vol. 34, article 44, 2015.

[98] R. Pallini, L. Ricci-Vitiani, G. L. Banna et al., "Cancer stem cell analysis and clinical outcome in patients with glioblastoma multiforme," Clinical Cancer Research, vol. 14, no. 24, pp. 8205$8212,2008$.

[99] C. Lottaz, D. Beier, K. Meyer et al., "Transcriptional profiles of $\mathrm{CD}_{133^{+}}$and $\mathrm{CD}_{133^{-}}$glioblastoma-derived cancer stem cell lines suggest different cells of origin," Cancer Research, vol. 70, no. 5, pp. 2030-2040, 2010.

[100] U. R. Chandran, S. Luthra, L. Santana-Santos et al., "Gene expression profiling distinguishes proneural glioma stem cells from mesenchymal glioma stem cells," Genomics Data, vol. 5, pp. 333-336, 2015.

[101] I. Saga, S. Shibao, J. Okubo et al., "Integrated analysis identifies different metabolic signatures for tumor-initiating cells in a murine glioblastoma model," Neuro-Oncology, vol. 16, no. 8, pp. 1048-1056, 2014.

[102] M. Meyer, J. Reimand, X. Lan et al., "Single cell-derived clonal analysis of human glioblastoma links functional and genomic heterogeneity," Proceedings of the National Academy of Sciences of the United States of America, vol. 112, no. 3, pp. 851-856, 2015.

[103] B. B. Endaya, P. Y. P. Lam, A. C. B. Meedeniya, and J. Neuzil, "Transcriptional profiling of dividing tumor cells detects intratumor heterogeneity linked to cell proliferation in a brain tumor model," Molecular Oncology, vol. 10, no. 1, pp. 126-137, 2016.

[104] M. Sugimori, Y. Hayakawa, B. M. Boman et al., "Discovery of power-law growth in the self-renewal of heterogeneous glioma stem cell populations," PLoS ONE, vol. 10, no. 8, Article ID e0135760, 2015.

[105] K. Christensen, H. D. Schrøder, and B. W. Kristensen, "CD133 niches and single cells in glioblastoma have different phenotypes," Journal of Neuro-Oncology, vol. 104, no. 1, pp. 129-143, 2011.

[106] R. K. Jain, E. di Tomaso, D. G. Duda, J. S. Loeffler, A. G. Sorensen, and T. T. Batchelor, "Angiogenesis in brain tumours," Nature Reviews Neuroscience, vol. 8, no. 8, pp. 610-622, 2007.

[107] P. Carmeliet and R. K. Jain, "Angiogenesis in cancer and other diseases," Nature, vol. 407, no. 6801, pp. 249-257, 2000.

[108] I. V. Ulasov, S. Nandi, M. Dey, A. M. Sonabend, and M. S. Lesniak, "Inhibition of sonic hedgehog and notch pathways enhances sensitivity of $\mathrm{CD}_{133^{+}}$glioma stem cells to temozolomide therapy," Molecular Medicine, vol. 17, no. 1-2, pp. 103-112, 2011.

[109] G.-N. Yan, L. Yang, Y.-F. Lv et al., "Endothelial cells promote stem-like phenotype of glioma cells through activating the Hedgehog pathway," Journal of Pathology, vol. 234, no. 1, pp. 1122, 2014.

[110] O. J. Becher, D. Hambardzumyan, E. I. Fomchenko et al., "Gli activity correlates with tumor grade in platelet-derived growth factor-induced gliomas," Cancer Research, vol. 68, no. 7, pp. 2241-2249, 2008. 
[111] K. Toyoda, K. Tanaka, S. Nakagawa et al., "Initial contact of glioblastoma cells with existing normal brain endothelial cells strengthen the barrier function via fibroblast growth factor 2 secretion: a new in vitro blood-brain barrier model," Cellular and Molecular Neurobiology, vol. 33, no. 4, pp. 489-501, 2013.

[112] P. Wang, H. Zhen, J. Zhang et al., "Survivin promotes glioma angiogenesis through vascular endothelial growth factor and basic fibroblast growth factor in vitro and in vivo," Molecular Carcinogenesis, vol. 51, no. 7, pp. 586-595, 2012.

[113] S. M. Pollard, K. Yoshikawa, I. D. Clarke et al., "Glioma stem cell lines expanded in adherent culture have tumor-specific phenotypes and are suitable for chemical and genetic screens," Cell Stem Cell, vol. 4, no. 6, pp. 568-580, 2009.

[114] K.-W. Chang, Y.-L. Huang, Z.-R. Wong et al., "Fibroblast growth factor-2 up-regulates the expression of nestin through the RasRaf-ERK-Sp1 signaling axis in C6 glioma cells," Biochemical and Biophysical Research Communications, vol. 434, no. 4, pp. 854860, 2013.

[115] G. Li, Z. Chen, Y.-D. Hu et al., "Autocrine factors sustain glioblastoma stem cell self-renewal," Oncology Reports, vol. 21, no. 2, pp. 419-424, 2009.

[116] E. M. Haley and Y. Kim, "The role of basic fibroblast growth factor in glioblastoma multiforme and glioblastoma stem cells and in their in vitro culture," Cancer Letters, vol. 346, no. 1, pp. $1-5,2014$.

[117] A. Pietras, A. M. Katz, E. J. Ekström et al., "Osteopontin-CD44 signaling in the glioma perivascular niche enhances cancer stem cell phenotypes and promotes aggressive tumor growth," Cell Stem Cell, vol. 14, no. 3, pp. 357-369, 2014.

[118] S. R. Chirasani, A. Sternjak, P. Wend et al., "Bone morphogenetic protein-7 release from endogenous neural precursor cells suppresses the tumourigenicity of stem-like glioblastoma cells," Brain, vol. 133, no. 7, pp. 1961-1972, 2010.

[119] N. Barabutis and A. V. Schally, "Growth hormone-releasing hormone: extrapituitary effects in physiology and pathology," Cell Cycle, vol. 9, no. 20, pp. 4110-4116, 2010.

[120] H. Kiaris, I. Chatzistamou, A. G. Papavassiliou, and A. V. Schally, "Growth hormone-releasing hormone: not only a neurohormone," Trends in Endocrinology and Metabolism, vol. 22, no. 8, pp. 311-317, 2011.

[121] Z.-J. Liu, T. Shirakawa, Y. Li et al., "Regulation of Notch1 and Dll4 by vascular endothelial growth factor in arterial endothelial cells: implications for modulating arteriogenesis and angiogenesis," Molecular and Cellular Biology, vol. 23, no. 1, pp. 14-25, 2003.

[122] I. B. Lobov, R. A. Renard, N. Papadopoulos et al., "Delta-like ligand 4 (Dll4) is induced by VEGF as a negative regulator of angiogenic sprouting," Proceedings of the National Academy of Sciences of the United States of America, vol. 104, no. 9, pp. 32193224, 2007.

[123] N. Charles, T. Ozawa, M. Squatrito et al., "Perivascular nitric oxide activates notch signaling and promotes stem-like character in PDGF-induced glioma cells," Cell Stem Cell, vol. 6, no. 2, pp. 141-152, 2010.

[124] C. E. Eyler, Q. Wu, K. Yan et al., "Glioma stem cell proliferation and tumor growth are promoted by nitric oxide synthase-2," Cell, vol. 146, no. 1, pp. 53-66, 2011.

[125] M. Scharpfenecker, J. J. C. M. Kruse, D. Sprong, N. S. Russell, P. ten Dijke, and F. A. Stewart, "Ionizing radiation shifts the PAI-1/ID-1 balance and activates notch signaling in endothelial cells," International Journal of Radiation Oncology, Biology, Physics, vol. 73, no. 2, pp. 506-513, 2009.
[126] Y.-F. Ping, X. Zhang, and X.-W. Bian, "Cancer stem cells and their vascular niche: do they benefit from each other?" Cancer Letters, 2015.

[127] Q. Shen, Y. Wang, E. Kokovay et al., "Adult SVZ stem cells lie in a vascular niche: a quantitative analysis of niche cell-cell interactions," Cell Stem Cell, vol. 3, no. 3, pp. 289-300, 2008.

[128] P. Huang, M. R. S. Rani, M. S. Ahluwalia et al., "Endothelial expression of TNF receptor-1 generates a proapoptotic signal inhibited by integrin $\alpha 6 \beta 1$ in glioblastoma," Cancer Research, vol. 72, no. 6, pp. 1428-1437, 2012.

[129] X. Zheng, Q. Xie, S. Li, and W. Zhang, "CXCR4-positive subset of glioma is enriched for cancer stem cells," Oncology Research, vol. 19, no. 12, pp. 555-561, 2012.

[130] R. Würth, A. Bajetto, J. K. Harrison, F. Barbieri, and T. Florio, "CXCL12 modulation of CXCR4 and CXCR7 activity in human glioblastoma stem-like cells and regulation of the tumor microenvironment," Frontiers in Cellular Neuroscience, vol. 8, article 144, 2014.

[131] D. Hambardzumyan, O. J. Becher, M. K. Rosenblum, P. P. Pandolfi, K. Manova-Todorova, and E. C. Holland, "PI3K pathway regulates survival of cancer stem cells residing in the perivascular niche following radiation in medulloblastoma in vivo," Genes \& Development, vol. 22, no. 4, pp. 436-448, 2008.

[132] W. B. Saunders, B. L. Bohnsack, J. B. Faske et al., "Coregulation of vascular tube stabilization by endothelial cell TIMP-2 and pericyte TIMP-3," The Journal of Cell Biology, vol. 175, no. 1, pp. 179-191, 2006.

[133] P. C. Stapor, R. S. Sweat, D. C. Dashti, A. M. Betancourt, and W. L. Murfee, "Pericyte dynamics during angiogenesis: new insights from new identities," Journal of Vascular Research, vol. 51, no. 3, pp. 163-174, 2014.

[134] J. T. Durham, H. K. Surks, B. M. Dulmovits, and I. M. Herman, "Pericyte contractility controls endothelial cell cycle progression and sprouting: insights into angiogenic switch mechanics," The American Journal of Physiology-Cell Physiology, vol. 307, no. 9, pp. C878-C892, 2014.

[135] A. L. Ribeiro and O. K. Okamoto, "Combined effects of pericytes in the tumor microenvironment," Stem Cells International, vol. 2015, Article ID 868475, 8 pages, 2015.

[136] Y. Soda, T. Marumoto, D. Friedmann-Morvinski et al., "Transdifferentiation of glioblastoma cells into vascular endothelial cells," Proceedings of the National Academy of Sciences of the United States of America, vol. 108, no. 11, pp. 4274-4280, 2011.

[137] M.-T. Chiao, Y.-C. Yang, W.-Y. Cheng, C.-C. Shen, and J.-L. Ko, "CD133+ glioblastoma stem-like cells induce vascular mimicry in vivo," Current Neurovascular Research, vol. 8, no. 3, pp. 210219, 2011.

[138] F. J. Rodriguez, B. A. Orr, K. L. Ligon, and C. G. Eberhart, "Neoplastic cells are a rare component in human glioblastoma microvasculature," Oncotarget, vol. 3, no. 1, pp. 98-106, 2012.

[139] S. El Hallani, B. Boisselier, F. Peglion et al., "A new alternative mechanism in glioblastoma vascularization: tubular vasculogenic mimicry," Brain, vol. 133, no. 4, pp. 973-982, 2010.

[140] S. El Hallani, C. Colin, Y. El Houfi et al., "Tumor and endothelial cell hybrids participate in glioblastoma vasculature," BioMed Research International, vol. 2014, Article ID 827327, 9 pages, 2014.

[141] P.-P. Zheng, M. van der Weiden, P. J. van der Spek, A. J. P. E. Vincent, and J. M. Kros, "Intratumoral, not circulating, endothelial progenitor cells share genetic aberrations with glial tumor cells," Journal of Cellular Physiology, vol. 228, no. 7, pp. 1383-1390, 2013. 
[142] N. Jhaveri, T. C. Chen, and F. M. Hofman, "Tumor vasculature and glioma stem cells: contributions to glioma progression," Cancer Letters, 2014.

[143] J. M. Heddleston, Z. Li, R. E. McLendon, A. B. Hjelmeland, and J. N. Rich, "The hypoxic microenvironment maintains glioblastoma stem cells and promotes reprogramming towards a cancer stem cell phenotype," Cell Cycle, vol. 8, no. 20, pp. 3274$3284,2009$.

[144] S. M. Wood, J. M. Gleadle, C. W. Pugh, O. Hankinson, and P. J. Ratcliffe, "The role of the aryl hydrocarbon receptor nuclear translocator (ARNT) in hypoxie induction of gene expression: studies in arnt-deficient cells," The Journal of Biological Chemistry, vol. 271, no. 25, pp. 15117-15123, 1996.

[145] L. Holmquist-Mengelbier, E. Fredlund, T. Löfstedt et al., "Recruitment of HIF- $1 \alpha$ and HIF- $2 \alpha$ to common target genes is differentially regulated in neuroblastoma: HIF- $2 \alpha$ promotes an aggressive phenotype," Cancer Cell, vol. 10, no. 5, pp. 413-423, 2006.

[146] G. Peng and Y. Liu, "Hypoxia-inducible factors in cancer stem cells and inflammation," Trends in Pharmacological Sciences, vol. 36, no. 6, pp. 374-383, 2015.

[147] L. Yang, C. Lin, L. Wang, H. Guo, and X. Wang, "Hypoxia and hypoxia-inducible factors in glioblastoma multiforme progression and therapeutic implications," Experimental Cell Research, vol. 318, no. 19, pp. 2417-2426, 2012.

[148] K. Takahashi, K. Tanabe, M. Ohnuki et al., "Induction of pluripotent stem cells from adult human fibroblasts by defined factors," Cell, vol. 131, no. 5, pp. 861-872, 2007.

[149] K. L. Covello, J. Kehler, H. Yu et al., "HIF- $2 \alpha$ regulates Oct-4: effects of hypoxia on stem cell function, embryonic development, and tumor growth," Genes and Development, vol. 20, no. 5, pp. 557-570, 2006.

[150] V. Moreno-Manzano, F. J. Rodríguez-Jiménez, J. L. AceñaBonilla et al., "FM19G11, a new hypoxia-inducible factor (HIF) modulator, affects stem cell differentiation status," The Journal of Biological Chemistry, vol. 285, no. 2, pp. 1333-1342, 2010.

[151] J. D. Gordan, J. A. Bertout, C.-J. Hu, J. A. Diehl, and M. C. Simon, "HIF- $2 \alpha$ promotes hypoxic cell proliferation by enhancing c-myc transcriptional activity," Cancer Cell, vol. 11, no. 4, pp. 335-347, 2007.

[152] M. Toda, "Glioma stem cells and immunotherapy for the treatment of malignant gliomas," ISRN Oncology, vol. 2013, Article ID 673793, 13 pages, 2013.

[153] K. De Bock, M. Mazzone, and P. Carmeliet, "Antiangiogenic therapy, hypoxia, and metastasis: risky liaisons, or not?" Nature Reviews Clinical Oncology, vol. 8, no. 7, pp. 393-404, 2011.

[154] M. Pàez-Ribes, E. Allen, J. Hudock et al., "Antiangiogenic therapy elicits malignant progression of tumors to increased local invasion and distant metastasis," Cancer Cell, vol. 15, no. 3, pp. 220-231, 2009.

[155] T. K. Mackey, R. Cuomo, C. Guerra, and B. A. Liang, "After counterfeit Avastin - what have we learned and what can be done?" Nature Reviews Clinical Oncology, vol. 12, no. 5, pp. 302308, 2015.

[156] R. Du, K. V. Lu, C. Petritsch et al., "HIFl $\alpha$ induces the recruitment of bone marrow-derived vascular modulatory cells to regulate tumor angiogenesis and invasion," Cancer Cell, vol. 13, no. 3, pp. 206-220, 2008.

[157] A. Soeda, M. Park, D. Lee et al., "Hypoxia promotes expansion of the CD133-positive glioma stem cells through activation of

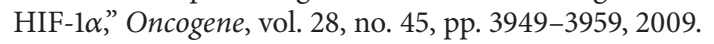

[158] E. E. Bar, A. Lin, V. Mahairaki, W. Matsui, and C. G. Eberhart, "Hypoxia increases the expression of stem-cell markers and promotes clonogenicity in glioblastoma neurospheres," The American Journal of Pathology, vol. 177, no. 3, pp. 1491-1502, 2010.

[159] X. Fan, L. Khaki, T. S. Zhu et al., "NOTCH pathway blockade depletes CD133-positive glioblastoma cells and inhibits growth of tumor neurospheres and xenografts," STEM CELLS, vol. 28, no. 1, pp. 5-16, 2010.

[160] L. Qiang, T. Wu, H.-W. Zhang et al., "HIF-1 $\alpha$ is critical for hypoxia-mediated maintenance of glioblastoma stem cells by activating Notch signaling pathway," Cell Death and Differentiation, vol. 19, no. 2, pp. 284-294, 2012.

[161] J. M. Heddleston, Q. Wu, M. Rivera et al., "Hypoxia-induced mixed-lineage leukemia 1 regulates glioma stem cell tumorigenic potential," Cell Death and Differentiation, vol. 19, no. 3, pp. 428-439, 2012.

[162] S. Zhao, Y. Lin, W. Xu et al., "Glioma-derived mutations in IDH1 dominantly inhibit IDH1 catalytic activity and induce HIF-1 $\alpha$," Science, vol. 324, no. 5924, pp. 261-265, 2009.

[163] A. B. Hjelmeland, Q. Wu, J. M. Heddleston et al., "Acidic stress promotes a glioma stem cell phenotype," Cell Death and Differentiation, vol. 18, no. 5, pp. 829-840, 2011.

[164] J. Chiche, M. C. Brahimi-Horn, and J. Pouysségur, “Tumour hypoxia induces a metabolic shift causing acidosis: a common feature in cancer," Journal of Cellular and Molecular Medicine, vol. 14, no. 4, pp. 771-794, 2010.

[165] R. Kulshreshtha, M. Ferracin, S. E. Wojcik et al., "A microRNA signature of hypoxia," Molecular and Cellular Biology, vol. 27, no. 5, pp. 1859-1867, 2007.

[166] H. Hu and R. A. Gatti, "MicroRNAs: new players in the DNA damage response," Journal of Molecular Cell Biology, vol. 3, no. 3, pp. 151-158, 2011.

[167] P. Fasanaro, S. Greco, M. Lorenzi et al., "An integrated approach for experimental target identification of hypoxia-induced miR210," The Journal of Biological Chemistry, vol. 284, no. 50, pp. 35134-35143, 2009.

[168] G. Shen, X. Li, Y.-F. Jia, G. A. Piazza, and Y. Xi, "Hypoxiaregulated microRNAs in human cancer," Acta Pharmacologica Sinica, vol. 34, no. 3, pp. 336-341, 2013.

[169] C. Tanase, R. Albulescu, E. Codrici et al., "Circulating biomarker panels for targeted therapy in brain tumors," Future Oncology, vol. 11, no. 3, pp. 511-524, 2015.

[170] S. Hara, J. Hamada, C. Kobayashi, Y. Kondo, and N. Imura, "Expression and characterization of hypoxia-inducible factor (HIF)-3 $\alpha$ in human kidney: suppression of HIF-mediated gene expression by HIF-3 $\alpha$," Biochemical and Biophysical Research Communications, vol. 287, no. 4, pp. 808-813, 2001.

[171] R. Agrawal, P. Pandey, P. Jha, V. Dwivedi, C. Sarkar, and R. Kulshreshtha, "Hypoxic signature of microRNAs in glioblastoma: insights from small RNA deep sequencing," BMC Genomics, vol. 15, no. 1, article 686, 2014.

[172] S. I. Grivennikov, F. R. Greten, and M. Karin, "Immunity, inflammation, and cancer," Cell, vol. 140, no. 6, pp. 883-899, 2010.

[173] I. F. Parney, J. S. Waldron, and A. T. Parsa, "Flow cytometry and in vitro analysis of human glioma-associated macrophages. Laboratory investigation," Journal of Neurosurgery, vol. 110, no. 3, pp. 572-582, 2009.

[174] J. J. Watters, J. M. Schartner, and B. Badie, "Microglia function in brain tumors," Journal of Neuroscience Research, vol. 81, no. 3, pp. 447-455, 2005. 
[175] W. Roggendorf, S. Strupp, and W. Paulus, "Distribution and characterization of microglia/macrophages in human brain tumors," Acta Neuropathologica, vol. 92, no. 3, pp. 288-293, 1996.

[176] L. Yi, H. Xiao, M. Xu et al., "Glioma-initiating cells: a predominant role in microglia/macrophages tropism to glioma," Journal of Neuroimmunology, vol. 232, no. 1-2, pp. 75-82, 2011.

[177] S.-C. Wang, J.-H. Hong, C. Hsueh, and C.-S. Chiang, “Tumorsecreted SDF-1 promotes glioma invasiveness and TAM tropism toward hypoxia in a murine astrocytoma model," Laboratory Investigation, vol. 92, no. 1, pp. 151-162, 2012.

[178] M. Tafani, M. Di Vito, A. Frati et al., "Pro-inflammatory gene expression in solid glioblastoma microenvironment and in hypoxic stem cells from human glioblastoma," Journal of Neuroinflammation, vol. 8, article 32, 2011.

[179] K. Gabrusiewicz, A. Ellert-Miklaszewska, M. Lipko, M. Sielska, M. Frankowska, and B. Kaminska, "Characteristics of the alternative phenotype of microglia/macrophages and its modulation in experimental gliomas," PLOS ONE, vol. 6, no. 8, Article ID e23902, 2011.

[180] E. Tartour, H. Pere, B. Maillere et al., "Angiogenesis and immunity: a bidirectional link potentially relevant for the monitoring of antiangiogenic therapy and the development of novel therapeutic combination with immunotherapy," Cancer and Metastasis Reviews, vol. 30, no. 1, pp. 83-95, 2011.

[181] J. M. Weiss, L. A. Ridnour, T. Back et al., "Macrophage-dependent nitric oxide expression regulates tumor cell detachment and metastasis after IL-2/anti-CD40 immunotherapy," The Journal of Experimental Medicine, vol. 207, no. 11, pp. 2455-2467, 2010.

[182] R. Albulescu, E. Codrici, I. D. Popescu et al., "Cytokine patterns in brain tumour progression," Mediators of Inflammation, vol. 2013, Article ID 979748, 7 pages, 2013.

[183] Q. Lahmar, J. Keirsse, D. Laoui, K. Movahedi, E. Van Overmeire, and J. A. Van Ginderachter, "Tissue-resident versus monocyte-derived macrophages in the tumor microenvironment," Biochimica et Biophysica Acta (BBA)-Reviews on Cancer, 2015.

[184] A. L. Ribeiro Xavier, B. T. Kress, S. A. Goldman, J. R. Lacerda de Menezes, and M. Nedergaard, "A distinct population of microglia supports adult neurogenesis in the subventricular zone," The Journal of Neuroscience, vol. 35, no. 34, pp. 1184811861, 2015.

[185] W. Paulus, W. Roggendorf, and T. Kirchuer, "Ki-M1P as a marker for microglia and brain macrophages in routinely processed human tissues," Acta Neuropathologica, vol. 84, no. 5, pp. 538-544, 1992.

[186] M. B. Graeber, B. W. Scheithauer, and G. W. Kreutzberg, "Microglia in brain tumors," Glia, vol. 40, no. 2, pp. 252-259, 2002.

[187] W. Zhou, S. Q. Ke, Z. Huang et al., "Periostin secreted by glioblastoma stem cells recruits M2 tumour-associated macrophages and promotes malignant growth," Nature Cell Biology, vol. 17, no. 2, pp. 170-182, 2015.

[188] S. M. Pyonteck, L. Akkari, A. J. Schuhmacher et al., "CSF-1R inhibition alters macrophage polarization and blocks glioma progression," Nature Medicine, vol. 19, no. 10, pp. 1264-1272, 2013.

[189] W. C. Huang, W. H. Chiang, Y. H. Cheng et al., "Tumortropic monocyte-mediated delivery of echogenic polymer bubbles and therapeutic vesicles for chemotherapy of tumor hypoxia," Biomaterials, vol. 71, pp. 71-83, 2015.
[190] D. Hanahan and R. A. Weinberg, "Hallmarks of cancer: the next generation," Cell, vol. 144, no. 5, pp. 646-674, 2011.

[191] A. R. Safa, M. R. Saadatzadeh, A. A. Cohen-Gadol, K. E. Pollok, and K. Bijangi-Vishehsaraei, "Glioblastoma stem cells (GSCs) epigenetic plasticity and interconversion between differentiated non-GSCs and GSCs," Genes \& Diseases, vol. 2, no. 2, pp. 152163, 2015.

[192] M. S. Wicha, S. Liu, and G. Dontu, "Cancer stem cells: an old idea-a paradigm shift," Cancer Research, vol. 66, no. 4, pp. 1883-1890, 2006.

[193] A.-M. Enciu, E. Codrici, I. Popescu, S. Mihai, R. Albulescu, and C. Tanase, "Patents in cacer stem cells," Recent Patents on Biomarkers, vol. 5, no. 1, pp. 3-13, 2015.

[194] Q. Shen, S. K. Goderie, L. Jin et al., "Endothelial cells stimulate self-renewal and expand neurogenesis of neural stem cells," Science, vol. 304, no. 5675, pp. 1338-1340, 2004.

[195] M. Kioi, H. Vogel, G. Schultz, R. M. Hoffman, G. R. Harsh, and J. M. Brown, "Inhibition of vasculogenesis, but not angiogenesis, prevents the recurrence of glioblastoma after irradiation in mice," The Journal of Clinical Investigation, vol. 120, no. 3, pp. 694-705, 2010.

[196] J. M. Heddleston, M. Hitomi, M. Venere et al., "Glioma stem cell maintenance: the role of the microenvironment," Current Pharmaceutical Design, vol. 17, no. 23, pp. 2386-2401, 2011.

[197] C. Folkins, S. Man, P. Xu, Y. Shaked, D. J. Hicklin, and R. S. Kerbel, "Anticancer therapies combining antiangiogenic and tumor cell cytotoxic effects reduce the tumor stem-like cell fraction in glioma xenograft tumors," Cancer Research, vol. 67, no. 8, pp. 3560-3564, 2007.

[198] G. L. Semenza, "Hypoxia-inducible factors: mediators of cancer progression and targets for cancer therapy," Trends in Pharmacological Sciences, vol. 33, no. 4, pp. 207-214, 2012.

[199] Y. Xia, H.-K. Choi, and K. Lee, "Recent advances in hypoxiainducible factor (HIF)-1 inhibitors," European Journal of Medicinal Chemistry, vol. 49, pp. 24-40, 2012.

[200] A. Palazon, A. W. Goldrath, V. Nizet, and R. S. Johnson, "HIF transcription factors, inflammation, and immunity," Immunity, vol. 41, no. 4, pp. 518-528, 2014.

[201] S. C. Cheng, J. Quintin, R. A. Cramer et al., "mTOR- and HIF$1 \alpha$-mediated aerobic glycolysis as metabolic basis for trained immunity," Science, vol. 345, no. 6204, Article ID 1250684, 2014.

[202] S. Saeed, J. Quintin, H. H. D. Kerstens et al., "Epigenetic programming of monocyte-to-macrophage differentiation and trained innate immunity," Science, vol. 345 , no. 6204, Article ID 1251086, 2014.

[203] S. Coma, A. Shimizu, and M. Klagsbrun, "Hypoxia induces tumor and endothelial cell migration in a semaphorin 3Fand VEGF-dependent manner via transcriptional repression of their common receptor neuropilin 2," Cell Adhesion and Migration, vol. 5, no. 3, pp. 266-275, 2011.

[204] F. Pistollato, S. Abbadi, E. Rampazzo et al., "Intratumoral hypoxic gradient drives stem cells distribution and MGMT expression in glioblastoma," STEM CELLS, vol. 28, no. 5, pp. 851-862, 2010.

[205] D. Tseng, D. A. Vasquez-Medrano, and J. M. Brown, “Targeting SDF-1/CXCR4 to inhibit tumour vasculature for treatment of glioblastomas," British Journal of Cancer, vol. 104, no. 12, pp. 1805-1809, 2011.

[206] M. Aubry, M. de Tayrac, A. Etcheverry et al., "From the core to beyond the margin: a genomic picture of glioblastoma intratumor heterogeneity," Oncotarget, vol. 6, no. 14, pp. 12094-12109, 2015. 
[207] K. Petrecca, M.-C. Guiot, V. Panet-Raymond, and L. Souhami, "Failure pattern following complete resection plus radiotherapy and temozolomide is at the resection margin in patients with glioblastoma," Journal of Neuro-Oncology, vol. 111, no. 1, pp. 1923, 2013.

[208] J. Lemée, A. Clavreul, and P. Menei, "Intratumoral heterogeneity in glioblastoma: don't forget the peritumoral brain zone," NeuroOncology, vol. 17, no. 10, pp. 1322-1332, 2015.

[209] S. J. Madsen, C. Christie, S. J. Hong et al., "Nanoparticleloaded macrophage-mediated photothermal therapy: potential for glioma treatment," Lasers in Medical Science, vol. 30, no. 4, pp. 1357-1365, 2015.

[210] S. J. Madsen, H. M. Gach, S. J. Hong, F. A. Uzal, Q. Peng, and H. Hirschberg, "Increased nanoparticle-loaded exogenous macrophage migration into the brain following PDT-induced blood-brain barrier disruption," Lasers in Surgery and Medicine, vol. 45, no. 8, pp. 524-532, 2013.

[211] J. Dong and Q. Huang, “Targeting glioma stem cells: enough to terminate gliomagenesis?” Chinese Medical Journal, vol. 124, no. 17, pp. 2756-2763, 2011. 

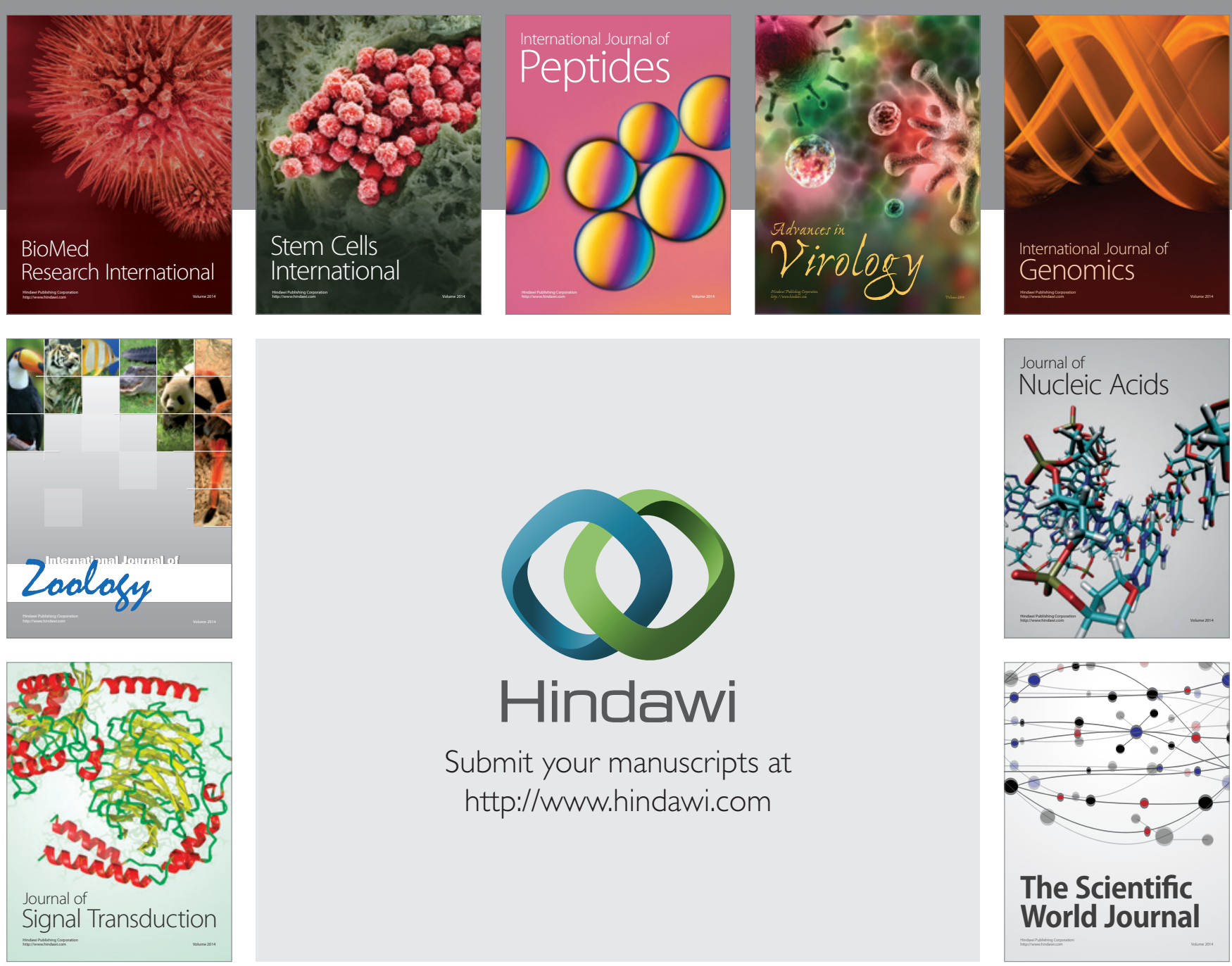

Submit your manuscripts at

http://www.hindawi.com
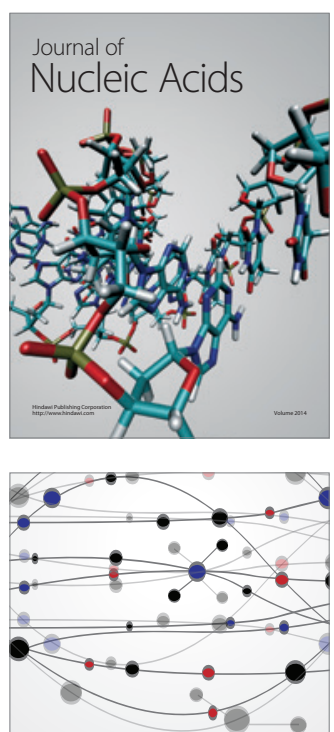

The Scientific World Journal
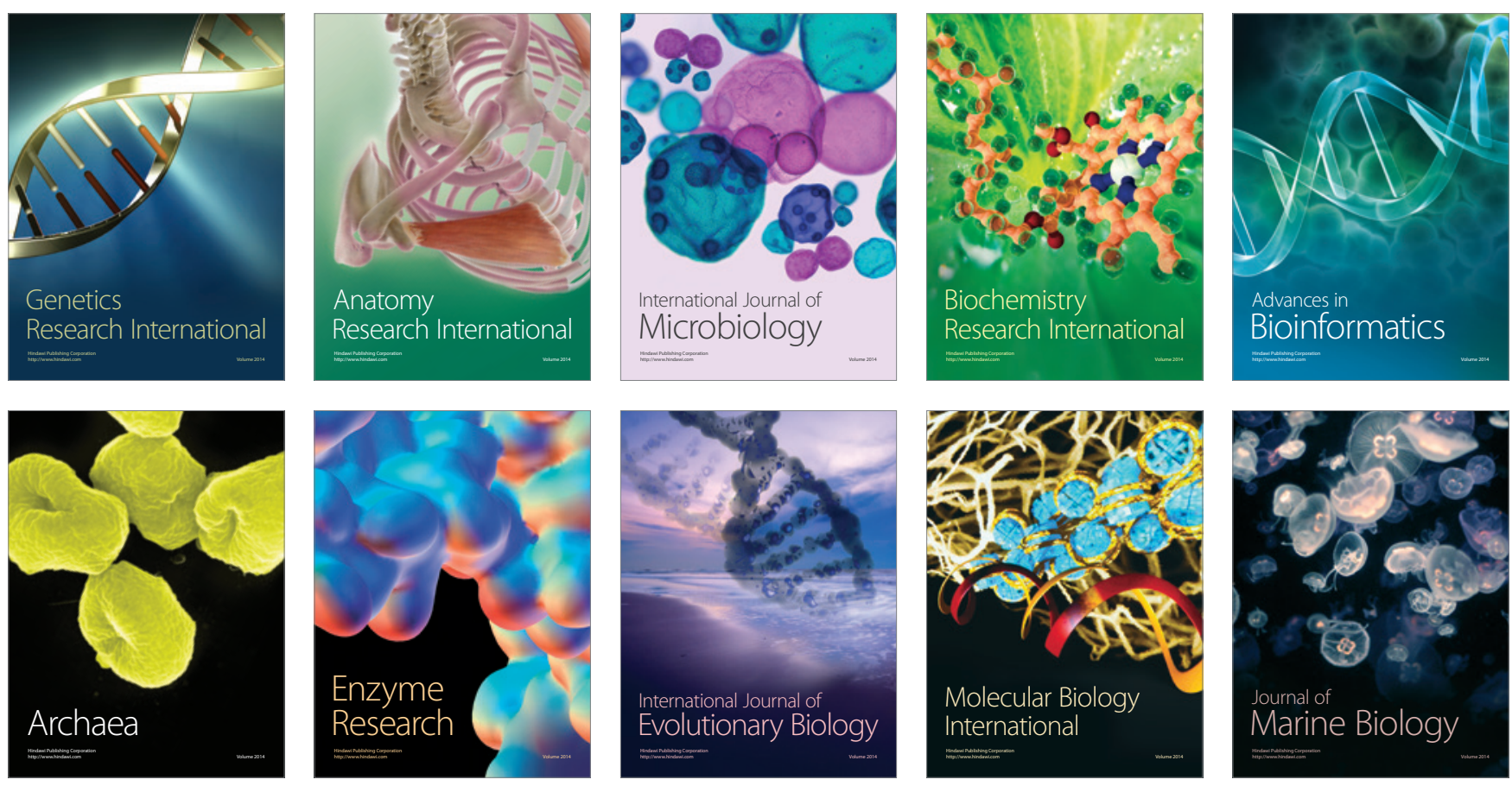